Linköping studies in science and technology. Thesis.

No. 1762

Licentiate's Thesis

\title{
Sampling Based Motion \\ Planning for Heavy Duty \\ Autonomous Vehicles
}

Niclas Evestedt

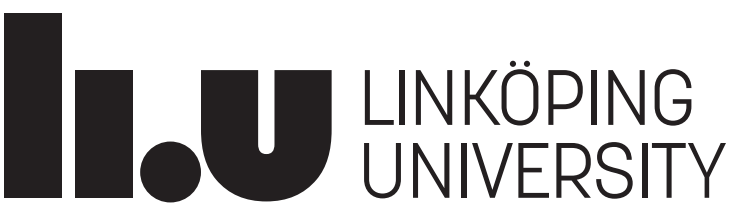


This is a Swedish Licentiate's Thesis.

Swedish postgraduate education leads to a Doctor's degree and/or a Licentiate's degree.

A Doctor's Degree comprises 240 ECTS credits (4 years of full-time studies).

A Licentiate's degree comprises 120 ECTS credits,

of which at least 60 ECTS credits constitute a Licentiate's thesis.

Linköping studies in science and technology. Thesis.

No. 1762

Sampling Based Motion Planning for Heavy Duty Autonomous Vehicles

Niclas Evestedt

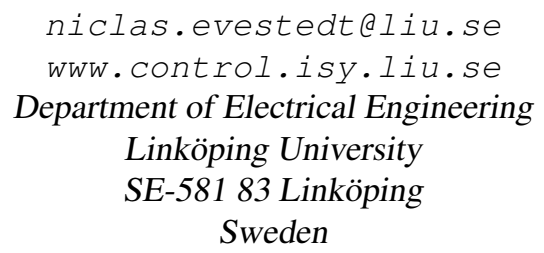

Sweden

ISBN 978-91-7685-667-3

ISSN 0280-7971

Copyright @ 2016 Niclas Evestedt

Printed by LiU-Tryck, Linköping, Sweden 2016 
To our new robot overlords! 



\section{Abstract}

The automotive industry is undergoing a revolution where the more traditional mechanical values are replaced by an ever increasing number of Advanced Driver Assistance Systems (ADAS) where advanced algorithms and software development are taking a bigger role. Increased safety, reduced emissions and the possibility of completely new business models are driving the development and most automotive companies have started projects that aim towards fully autonomous vehicles. For industrial applications that provide a closed environment, such as mining facilities, harbors, agriculture and airports, full implementation of the technology is already available with increased productivity, reliability and reduced wear on equipment as a result. However, it also gives the opportunity to create a safer working environment when human drivers can be removed from dangerous working conditions. Regardless of the application an important part of any mobile autonomous system is the motion planning layer. In this thesis sampling-based motion planning algorithms are used to solve several non-holonomic and kinodynamic planning problems for car-like robotic vehicles in different application areas that all present different challenges.

First we present an extension to the probabilistic sampling-based Closed-Loop Rapidly exploring Random Tree (CL-RRT) framework that significantly increases the probability of drawing a valid sample for platforms with second order differential constraints. When a tree extension is found infeasible a new acceleration profile that tries to brings the vehicle to a full stop before the collision occurs is calculated. A resimulation of the tree extension with the new acceleration profile is then performed. The framework is tested on a heavy-duty Scania G480 mining truck in a simple constructed scenario.

Furthermore, we present two different driver assistance systems for the complicated task of reversing with a truck with a dolly-steered trailer. The first is a manual system where the user can easily construct a kinematically feasible path through a graphical user interface. The second is a fully automatic planner, based on the CL-RRT algorithm where only a start and goal position need to be provided. For both approaches, the internal angles of the trailer configuration are stabilized using a Linear Quadratic (LQ) controller and path following is achieved through a pure-pursuit control law. The systems are demonstrated on a small-scale test vehicle with good results.

Finally, we look at the planning problem for an autonomous vehicle in an urban setting with dense traffic for two different time-critical maneuvers, namely, intersection merging and highway merging. In these situations, a social interplay between drivers is often necessary in order to perform a safe merge. To model this interaction a prediction engine is developed and used to predict the future evolution of the complete traffic scene given our own intended trajectory. Real-time capabilities are demonstrated through a series of simulations with varying traffic densities. It is shown, in simulation, that the proposed method is capable of safe merging in much denser traffic compared to a base-line method where a constant velocity model is used for predictions. 



\section{Populärvetenskaplig sammanfattning}

Fordonsindustrin genomgår just nu en revolution där en rad avancerade förarstödsystem och tjänster introduceras vilket innebär att avancerad algoritm och mjukvaruutveckling får en allt viktigare roll. Ökad säkerhet, minskade utsläpp och möjligheten för helt nya affärsmodeller är drivande krafter bakom utvecklingen och de flesta större fordonstillverkare har startat projekt som siktar mot helt självkörande bilar där föraren är frånkopplad kontrollen av fordonet. Trafikolyckor är den globalt sett vanligaste dödsorsaken för människor mellan 15 och 29 år vilket motiverar utvecklingen av säkrare transportsystem men många forskningsfrågor kring rimligheten och effekterna av ett helt automatiserat transportsystem återstår.

För industriella applikationer så som gruvor, hamnar, jordbruk eller flygplatser där en avgränsad yta finns är möjligheterna för full implementation mycket närmare och finns redan idag implementerat på många ställen. Ökad produktivitet, tillgänglighet och minskat slitage på utrustning är några av vinsterna man ser men även möjligheten att flytta människor från farliga arbetsmiljöer, som tex underjordsgruvor där risken för olyckor är större, till kontrollcentraler ovan jord där maskiner kan fjärrstyras eller en automatiserad process kan övervakas.

Oavsett applikationen så är möjligheten att effektivt planera och utföra säkra förflyttningar av ett robotiserat fordon en viktig del av ett autonomt system. I den här avhandlingen använder vi oss av stickprovsbaserad rörelseplanering för icke-holonomiska och kinodynamiska planeringsproblem för en rad olika applikationer som alla har olika utmaningar.

Först presenterar vi en vidareutveckling av den sannolikhetsbaserade stickprovsalgoritmen Rapidly exploring Random Tree (RRT) där vi utvecklar en metod där vi genom att använda information från flera simuleringar av systemet kan beräkna en giltig hastighetsprofil som kan användas för att rätta till ett misslyckat stickprov. Denna metod ökar markant sannolikheten för att dra ett stickprov som genererar en giltig trajektoria i kritiska lägen. Systemet har sedan testats på en lastbil anpassad för gruvapplikationer.

Vi presenterar även två olika förarstödsystem för att förenkla den komplicerade uppgiften att backa med en lastbil med tungt släp. Först visar vi ett manuellt system där en förare enkelt kan planera en kinematiskt korrekt bana genom att använda ett grafiskt användargränssnitt och sedan ett annat system med helt automatiserad planering av en bana från en startpunkt till en slutpunkt med hjälp av RRT. Dessa system testades sedan på en småskalig testplattform med goda resultat.

Slutligen studerades ett planeringsproblem för att hantera korsningar och påfarter på motorväg i tät trafik där ett samspel mellan förare ofta är nödvändigt för att genomföra en manöver. En prediktionsmotor baserad på smarta förarmodeller används för att prediktera hur hela trafikscenen utvecklas framåt i tiden beroende på vår egen trajektoria. Genom diskretisering och snabba uträkningar av flera lämpliga hastighetsprofiler som kan utvärderas genom prediktionsmotorn så kan en slutgiltigt trajektoria för det egna fordonet bestämmas. 



\section{Acknowledgments}

First of all, I would like to give a special thanks to my supervisor Assoc. Prof. Daniel Axehill for his support and encouragement throughout this work. He has a genuine interest in what we do and his door is always open for discussions and new ideas. Without his support this thesis would most likely not have been finished, probably not even started. I would also like to thank Oskar Ljungqvist and Erik Ward for the fantastic collaboration and fruitful discussions we have had during the last years.

I would also like to show my gratitude to my co-supervisor Prof. Fredrik Gustafsson and Prof. Svante Gunnarsson for inviting me to be part of the Automatic Control group. I am also very grateful for their help and flexibility when arranging a possibility to finish this thesis even after I moved away from Linköping. Thanks to Ninna Stensgård for making sure everything runs smoothly and for the help with all practical matters. I would also like to acknowledge FFI/VINNOVA and the iQMatic project for the financial support. I also appreciate the great work and help from Gustaf Hendeby regarding Latex questions and for providing the template for this thesis. I appreciate all the great help and want to thank Robin Lilja, Oskar Ljungqvist, Hanna Nyqvist and Clas Veibäck for helping me proof read various parts of this thesis.

Before I started as a PhD student in Linköping I lived in Uppsala, a city that I really like, so it was a hard decision to take the step and move to a completely new city. One of my closest friends even warned me of the "social suicide" I would commit if I left Uppsala. However, he was completely wrong and I want to give a special thanks to all my colleagues at Automatic Control for all the fun we have had with everything from crazy discussions at the office to awesome parties and bar crawling. I have gained many new and amazing friends! A special thanks goes to Hanna Nyqvist, Sina Khoshfetrat Pakazad and André Bittencourt for their hospitality when letting me sleep on the amazing couch in their living room during my visits when I have been working in Linköping during the past year. My roommate and friend George Mathai deserves a special thanks for all interesting topics that have come up during our discussions and I hope your towel have found a better place now when you have moved to Germany.

Prof. Patric Jensfelt at Robotics, Perception and Learning (RPL) at the Royal Institute of Technology also needs a special thanks for organizing a place to work after I moved from Linköping. The colleges at RPL have always made me feel welcome. The iQMatic team at Scania also needs a special thanks for making the time I spent as an intern a great learning experience and a fun time.

Last but not least, I am most grateful for my family and their support and encouragement. Elisabeth, thank you for all your love and for withstanding living with me even though I sometimes have crazy plans and not always take the most straight forward path.

Linköping, October 2016

Niclas Evestedt 



\section{Contents}

\section{Background}

1 Introduction 3

1.1 Motivation . . . . . . . . . . . . . . . . . 3

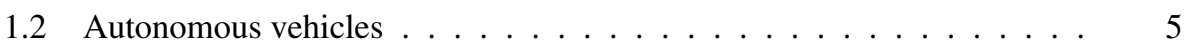

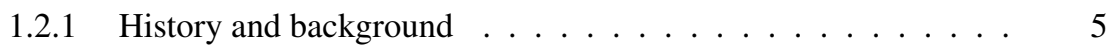

1.2.2 System architecture . . . . . . . . . . . . . . . . 7

1.3 Thesis outline . . . . . . . . . . . . . . . . . . . . . . . . . . . . . . .

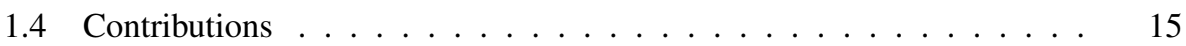

2 Classical motion planning 17

2.1 Configuration space . . . . . . . . . . . . . . . . . . 17

2.2 Popular approaches . . . . . . . . . . . . . . . . . . . . . . . . . . . . . . . 19

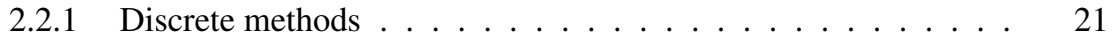

2.2.2 Probabilistic methods ............... 27

3 Motion planning for autonomous vehicles 33

3.1 Differential constraints . . . . . . . . . . . . . . 34

3.1.1 Two-point boundary value problem . . . . . . . . . . . 36

3.2 Planning under differential constraints . . . . . . . . . . . . . . 37

3.2 .1 Unstructured areas . . . . . . . . . . . . . . . . . . . . 38

3.2 .2 Structured areas . . . . . . . . . . . . 43

4 Concluding remarks 49

4.1 Summary of contributions . . . . . . . . . . . . . . . 49

4.2 Future work ......................... 50

$\begin{array}{ll}\text { Bibliography } & 53\end{array}$ 


\section{Publications}

A Sampling Recovery for Closed Loop Rapidly Expanding Random Tree using Brake Profile Regeneration

1 Introduction . . . . . . . . . . . . . . . . . . . 65

$1.1 \quad$ Related work . . . . . . . . . . . . . . . . . 66

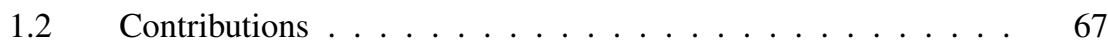

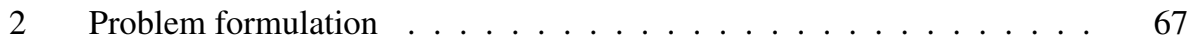

3 CL-RRT framework . . . . . . . . . . . . . . . 68

3.1 Tree expansion . . . . . . . . . . . . . . . 68

$3.2 \quad$ Safe states . . . . . . . . . . . . . . . . 68

3.3 Node connection heuristic . . . . . . . . . . . . . 69

3.4 Sampling strategy . . . . . . . . . . . . . . . . . 69

3.5 Online replanning . . . . . . . . . . . . . . . 70

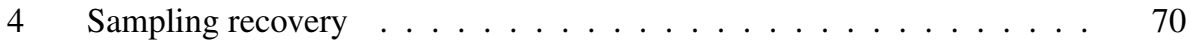

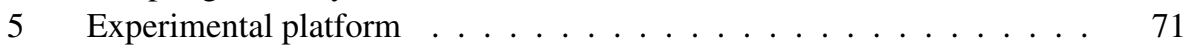

$5.1 \quad$ Vehicle model . . . . . . . . . . . . . . . 72

5.2 Vehicle controller . . . . . . . . . . . . . . 73

$6 \quad$ Results . . . . . . . . . . . . . . . . . . 74

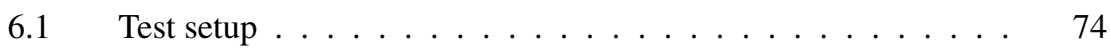

$6.2 \quad$ Parameter setup . . . . . . . . . . . . . . . . . . 74

6.3 Application results . . . . . . . . . . . . 75

7 Conclusions . . . . . . . . . . . . . . 76

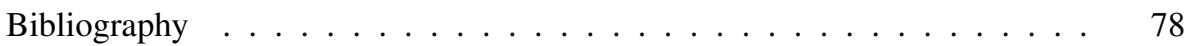

B Path tracking and stabilization for a reversing general 2-trailer configura$\begin{array}{ll}\text { tion using a cascaded control approach } & \mathbf{8 1}\end{array}$

1 Introduction . . . . . . . . . . . . . . . . . . . . 83

$1.1 \quad$ Related work . . . . . . . . . . . . . . . . . . . 84

2 System dynamics . . . . . . . . . . . . . . . . . 85

$2.1 \quad$ Linearization . . . . . . . . . . . . . . . . . . 86

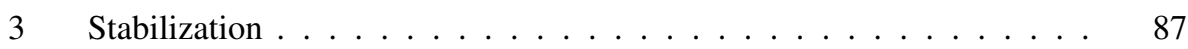

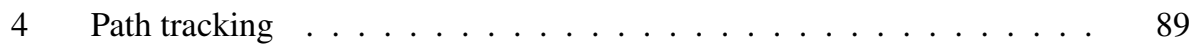

$4.1 \quad$ Stability . . . . . . . . . . . . . . . . . . 90

5 User interface . . . . . . . . . . . . . . . . . . . . . . . . . 90

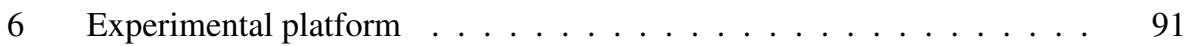

$6.1 \quad$ Parameters .................... 91

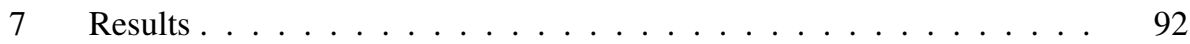

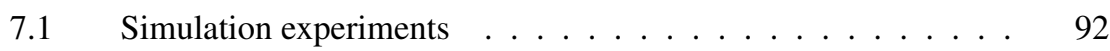

7.2 Lab experiments . . . . . . . . . . . . . . . . . . . . . . . . 92

8 Conclusions and future work . . . . . . . . . . . . . . . . . 94

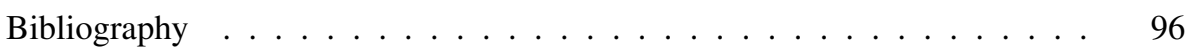

C Motion planning for a reversing general 2-trailer configuration using Closed$\begin{array}{lr}\text { Loop RRT } & 99\end{array}$

1 Introduction . . . . . . . . . . . . . . . . . 101 
$1.1 \quad$ Related work . . . . . . . . . . . . . . . . . 102

2 RRT-framework . . . . . . . . . . . . . . . . . . . 104

$2.1 \quad$ Tree expansion . . . . . . . . . . . . . . . . 104

3 System dynamics . . . . . . . . . . . . . . . . . . . . . . . 106

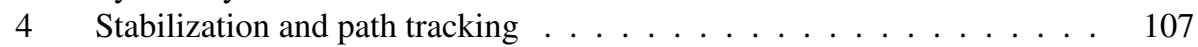

4.1 LQ-controller . . . . . . . . . . . . . . . . . 107

4.2 Path tracking . . . . . . . . . . . . . . . . 108

5 RRT-integration . . . . . . . . . . . . . . . . . . . . . 109

$5.1 \quad$ Node connection heuristic . . . . . . . . . . . . . 109

5.2 Goal evaluation . . . . . . . . . . . . . . . . . . . 110

5.3 Cost function . . . . . . . . . . . . . . . 110

6 Experimental platform ...................... 111

$6.1 \quad$ Parameters ....................... 111

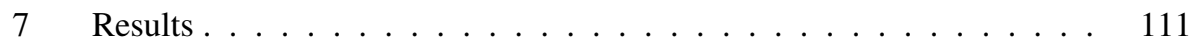

$7.1 \quad$ Maze . . . . . . . . . . . . . . . . 112

7.2 Three point turn . . . . . . . . . . . . . 113

$7.3 \quad$ Driver test . . . . . . . . . . . . . . . . 114

7.4 Real world ....................... 116

8 Conclusions and future work . . . . . . . . . . . . . 116

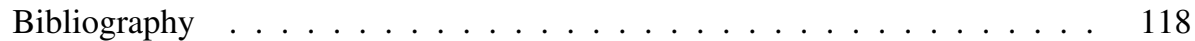

D Interaction aware trajectory planning for merge scenarios in congested traffic situations

1 Introduction . . . . . . . . . . . . . . . . . . . 124

$1.1 \quad$ Related work . . . . . . . . . . . . . . . . . . 125

2 Interaction aware motion planning . . . . . . . . . . . . . 126

2.1 Trajectory generation . . . . . . . . . . . . . . . . . . 127

2.2 Prediction engine . . . . . . . . . . . . . . . . . 129

2.3 Cost function . . . . . . . . . . . . . . . . . 130

3 Simulations ............................ 132

$3.1 \quad$ Simulation setup . . . . . . . . . . . . . . . . . 133

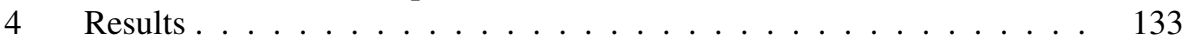

$4.1 \quad$ Statistical Evaluation . . . . . . . . . . . . . . 135

5 Conclusions and future work . . . . . . . . . . . . . . . 137

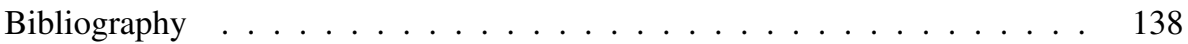



Part I

\section{Background}





\section{1}

\section{Introduction}

This chapter will introduce the reader to the history and the general research field of autonomous vehicles and advanced driver assistance system (ADAS) and give a motivation of why it is important to focus attention in this fast moving field. An overview of the iQMatic project in which this work has been performed will be given together with the contributions and finally, the outline of the rest of this thesis.

\subsection{Motivation}

In the past decades the automotive industry has seen a rapid development from being mostly focused on mechanical engineering to now where most companies are seeing their future in advanced high-technological features and services and therefore are shifting attention towards more advanced software development within their platforms. Increased safety, reduced emissions and an overall better utilization of the available infrastructure are some of the driving forces and demands from society. Car manufacturers can on their side see an added value for their customers and the possibility to gain an edge on competitors by implementing this new technology. Cost effective transportation of both goods and people is one of the backbones in the modern economy, but increasing demand put a high pressure on existing infrastructure. Congestion, reduced air quality, increasing $\mathrm{CO}_{2}$ emissions and increasing accident rates are some of the problems that can be seen as a result. As the transportation sector is the biggest emitter of greenhouse gases, just after electricity production, these problems are taken seriously and the European Road Transport Research Advisory Council (2013) and European Comission (2011) want tough goals for the European transportation sector with an overall efficiency improvement of the transport system by $50 \%$ in 2030 compared to 2010 and increasing mobility and transportation throughput while reducing emissions by $60 \%$.

Apart from emission reductions and efficiency, safety is another concern. According to World Health Organization (2015) road traffic accidents claim more than 1.2 million 
lives annually and injures 40 to 50 million people globally and is the main cause of death of people aged between 15 to 29 years old. Dahdah and McMahon (2008) estimates that $3 \%$ of global GDP is lost due to road traffic related injuries and deaths. Electronic stability control and anti-lock braking systems are effective means for preventing and reducing the severity of accidents and are now mandatory by law for all newly produced cars in many high-income countries, however the implementation of these systems is lagging behind in less developed parts of the world (World Health Organization, 2015; Lie et al., 2006). While there is a huge potential globally to implement technology that already exists the development of more advanced ADAS systems is fast moving and recent developments in sensor technology, such as RADAR, LiDAR and camera algorithms give possibilities to detect and handle critical situations long before the average driver can react. Automatic braking systems are now mandatory for newly produced heavy goods vehicles within the European Union and recently the National Highway Traffic Safety Administration (2015a) announced the commitment of 20 automakers to fit all vehicles with automatic braking as a standard feature no later than 2022 on the US market. ADAS systems such as lane departure warning, lane keep assist, adaptive cruise control and queue assist are other features that are becoming more frequent in modern cars. These systems are getting more and more control of the vehicle and can relieve the driver in critical situations but also from stressful and sometimes boring tasks such as driving in queues on congested roads. With features such as parking assist where vehicles can perform automatic parallel parking and the well-known autopilot from Tesla (Mchug, 2015), fully autonomous vehicles are getting closer and have the potential to revolutionize the transport sector. With 94\% of accidents caused by driver error according to National Highway Traffic Safety Administration (2015b), fully autonomous vehicles are an attractive idea that not only has the potential to reduce accidents but also push the transportation sector towards a more resource efficient service based system. When cars are estimated to be parked and at stand still $95 \%$ of the time, enormous resources, both in terms of parking space and material used for every privately owned car, could be released by a combination of autonomous vehicles and smart ride sharing services. This would give much needed space to city planners to improve, not only the resource efficiency of the city but also make it more livable with more space for pedestrians and green areas (Morris, 2016).

Inner city driving presents a complex scene with pedestrians, cars, buses, bicycles etc. and robust autonomous performance in these areas is still a hard challenge even for the most advanced systems. This together with the legislation changes needed to operate on public roads, make mines, harbors, loading terminals, airports and other closed off areas with less complex scenes a perfect starting ground for full implementation of this technology. Increased productivity, higher up time, reduced emissions and lower wear on equipment are expected outcomes of the implementation but also the possibility of removing human workers from dangerous working conditions that are often present in e.g. underground mines. Commercial systems for these applications already exist and are in full operation but the wide global implementation of autonomous systems is only just beginning so huge market potential can be seen. To develop and secure competence for future development and increase collaboration between universities and industry in the area of autonomous vehicles the Vinnova funded iQMatic project was started in 2013 with both industry and university partners (KTH, LiU, Scania CV, Saab and Autoliv) with a common goal to develop a research platform capable of performing full scale autonomous 


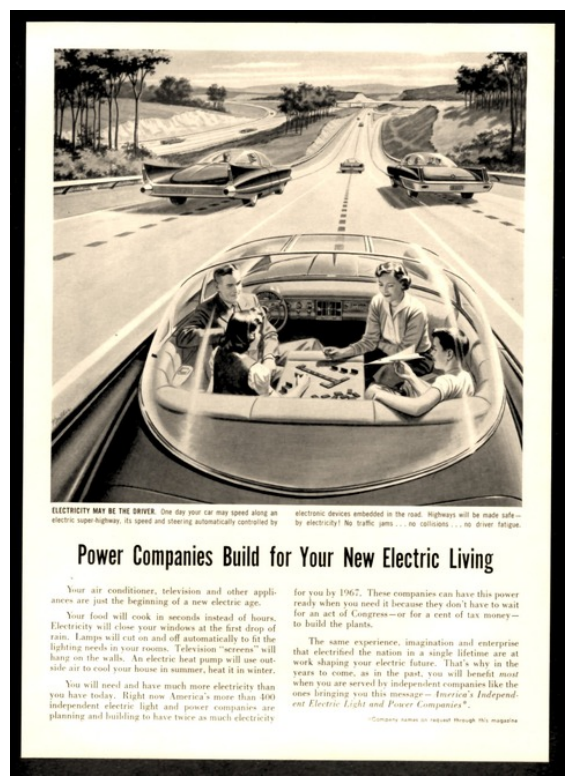

Figure 1.1: Advertisement for America's Electric Light and Power Companies in Saturday Evening Post, 1950s. A family plays a game while cruising autonomously down the highway in an autonomous vehicle.

operation at a typical mining facility with problems ranging from control room interfaces for operators to low level control of vehicles. This thesis has been done within this project and has the focus on motion planning algorithms for robotic vehicles.

\subsection{Autonomous vehicles}

This section first gives a brief background and history of the development of autonomous vehicles before a description of a common system architecture is given to get a better understanding of how this work fits in the bigger picture of an autonomous system.

\subsubsection{History and background}

The dream of autonomous or driverless cars is probably as old as the car itself. Autonomous cars have appeared in science fiction literature numerous times with some dating back to the early 1930s. General Motors presented their vision of the future city on the 1939 New York World's Fair, with over 40 million visitors, in the Futurama exhibition (Ferlis, 2007). A scale model, over $3000 \mathrm{~m}^{2}$ in size, of a 1960s city with automated highway systems seamlessly connecting all parts of the city could be viewed by visitors traveling on moving seats around the exhibition and in 1950s America's Electric Light and Power Companies were running an ad with a futuristic automated highway as seen in Figure 1.1. Now almost 80 years later we know that these visions never became reality 
and today's dreams of automated vehicles have changed little compared to the visions presented back then. However, compared to the available technology now and then the dream is much more achievable. With the digital revolution in the 1960s and the pioneering work in artificial intelligence by Moravec (1990), the first steps towards intelligent and autonomous robots where taken. However, it was not until the 1980s with the pioneering work of Dickmanns and Zapp (1987) were the first steps towards driverless cars capable of operating on normal roads were taken. With the Eureka PROMETHEUS Project (PROgraMme for a European Traffic of Highest Efficiency and Unprecedented Safety) the platform was further developed and cumulated with Dickmanns et al. (1994) work on a Mercedes 500 SEL that demonstrated autonomous driving during real conditions on a three lane highway in Paris with vision tracking of other vehicles, lane changes and lane tracking capabilities at speeds over $130 \mathrm{~km} / \mathrm{h}$. Carnegie Mellon University were at the same time experimenting with their Navlab platform and achieved basic autonomous operation on highways using a camera based vision system (Thorpe et al., 1988, 1991). Building on results from PROMETHEUS, Broggi et al. (1999) again demonstrated autonomous operation on highways over a $2000 \mathrm{~km}$ long track in Italy with the ARGO vehicle.

The groundbreaking Grand Challenges conducted in 2004 and 2005 that was sponsored by DARPA are often seen as the starting point of the intense efforts in autonomous driving we see today. The robots were presented with a previously unknown, over 200 $\mathrm{km}$ long, track through areas around the Mojave desert with difficult terrain and narrow gravel roads. A predefined GPS path was laid out and the vehicles had to navigate inside a driving corridor around the path at speeds up to $80 \mathrm{~km} / \mathrm{h}$ without any manual intervention while negotiating stones, bushes, fences, ditches and other obstacles present around the road. In the first challenge in 2004 none of the robots managed to finish the course but Carnegie Mellon University's vehicle Sandstorm managed to travel the farthest distance and completed $11.78 \mathrm{~km}$ of the course. A new challenge was announced for the next year and now four teams managed to complete the course and after a tight race Stanford made history and came out on top with their vehicle Stanley that finished the course in just under $7 \mathrm{~h}$ (Thrun et al., 2006; Whittaker, 2005; Trepagnier et al., 2005; Braid et al., 2006). To continue the efforts DARPA announced the Urban Challenge to be held in 2007 and now in a much more complicated setting. The competition was held in an old closed off air force base and vehicles had to obey the traffic rules and consider other robotic and human driven vehicles while negotiating intersections and urban environments on a 96 $\mathrm{km}$ long course. Carnegie Mellon University was determined as the winner with their vehicle BOSS and Stanford University finished second after a tight race where not only the finishing time was considered but also the behavior of the vehicles when negotiating intersections and other traffic situations (Urmson et al., 2008; Montemerlo et al., 2008).

Since the competitions, impressive progress has been made and several self-driving car projects have been started including probably the most famous being the Self Driving Car project at Google that so far has logged almost 2.5 million km of autonomous operation in various driving situations including dense inner city traffic with only minor incidents (Google, 2016). Other impressive efforts include the Bertha project by Mercedes in collaboration with Forschungszentrum Informatik and Karlsruhe Institute of Technology (Ziegler et al., 2014b), the Public Road Urban Driverless Car Test (PROUD) by University of Parma (Broggi et al., 2015) and the Stadtpilot project (Nothdurft et al., 2011). Building 


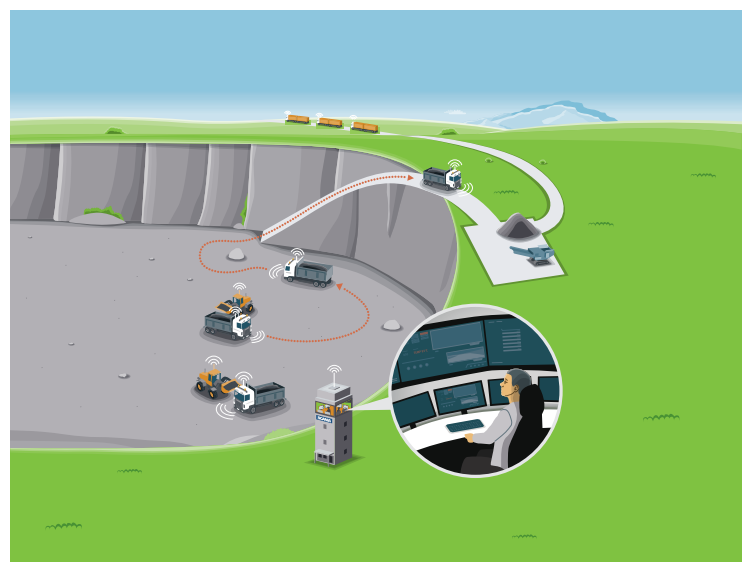

Figure 1.2: Illustration of a future mining facility. Connected and automated vehicles perform loading and transportation tasks while a human operator monitors the operation from a control tower. The image is used with courtesy of Scania AB.

on the success and inspiration from many of these projects almost all major car manufacturers have now launched projects involving autonomous vehicles and there is a race to be the first company with an autonomous car on the market. Many start-up companies are also focusing on the technology and are challenging the more developed brands on the market with new business models and innovative ideas that could change the way we use transportation services today.

\subsubsection{System architecture}

The goal of the iQMatic project is to develop a research and demonstration platform capable of performing transportation tasks at a typical mining facility. The vision of the project is illustrated in Figure 1.2 and spans all the way from control room interfaces to low-level vehicle controllers. System architectures for autonomous vehicles can vary depending on the task at hand, so to give the reader an understanding of how this work fits within a complete autonomous system, not limited to only mining, a typical architecture for an autonomous vehicle will be given in this section. The iQMatic platform follows this typical architecture but details can vary. Big systems are often divided into several modules that each is responsible for a different task and communication interfaces is then defined so relevant information can be communicated between modules. If implemented correctly this architecture gives a modular and scalable system where it is easy to test new approaches by just swapping out any given module. In Figure 1.3 an overview of the most important modules in a typical system is illustrated and the rest of this section will give some more background information and references for further reading about each module. 


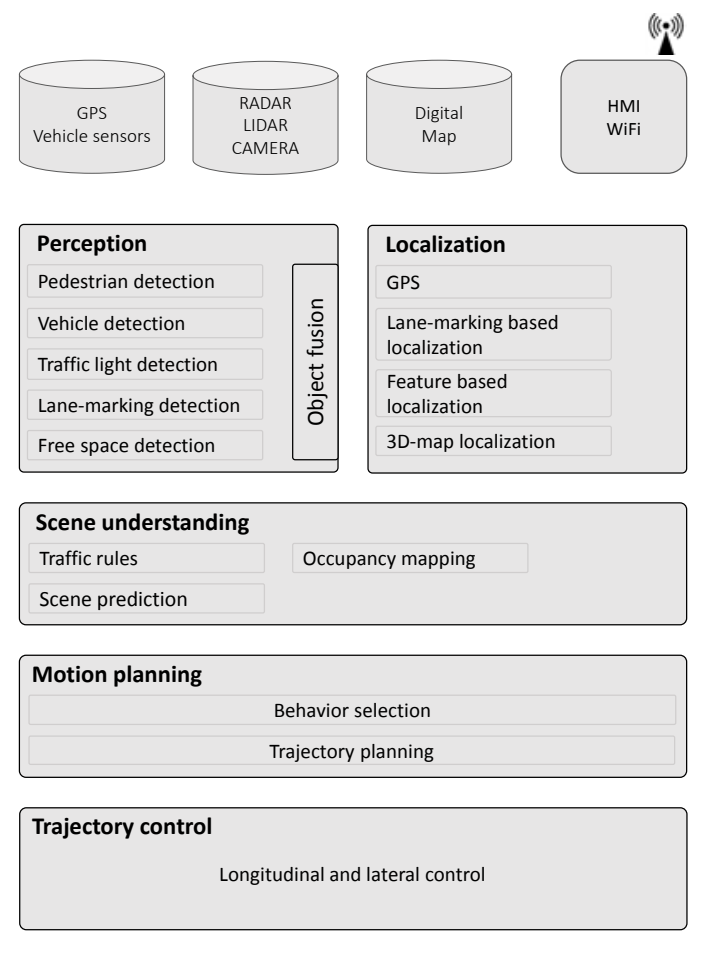

Figure 1.3: System overview of a typical architecture for an autonomous vehicle and the modules constituting a full system.

\section{Perception and localization}

The most common perception sensors used for autonomous vehicles are RADAR, LiDAR and camera sensors. All of these sensors have different pros and cons thus a fusion between data from all or combinations of these sensors are often seen in real systems. The task of the perception system is to detect, classify and track different objects such as pedestrians, traffic lights, other vehicles, lane markings, bicyclists and other static or dynamic obstacles in the environment around the vehicle. LiDAR was the main sensor choice for many teams during the DARPA Urban Challenge and many teams used the powerful 64 beam version of the spinning Velodyne laser scanner to get a $360^{\circ}$ field of view around the vehicle (Vel, 2013; Urmson et al., 2008; Montemerlo et al., 2008). LiDAR sensors are also the main sensor for perception and localization in the Google Self Driving car project (Google, 2016) and is also used extensively in the efforts by Ford and the University of Michigan (Ford, 2015).

Accurate localization is a crucial part of many autonomous systems, especially when lane markings are missing or in complex intersections where current perception technology cannot understand the context of an intersection by sensor data alone. GPS sensors 
are usually too unreliable in obstructed scenes with tall buildings and do not work at all in tunnels or when driving under over-hanging structures so other methods need to be used. To solve the localization problem, high precision 3D-maps are built by mapping the area in an offline run. Then by comparing current sensor data from the LiDAR to the 3D-map, accurate position estimates can be obtained (Wolcott and Eustice, 2015, 2014). Using recent advances in machine learning, LiDAR data is also used to detect and classify different objects around the vehicle by using hand labeled training data of pedestrians, vehicles, bicyclists etc. (Wang et al., 2012). However, these high performance LiDAR sensors are often considered too expensive for wide scale deployment and keeping consistent maps with changing weather, lighting and environment conditions is a hard and unsolved problem.

Other projects have focused on the much cheaper camera sensor as the main choice. With the breakthrough of deep Convolution Neural Networks (CNN) and specialized hardware for image classification, cameras have fast become superior in scene segmentation for urban scenarios and is often fused together with radar data for increased range and depth estimation (Krizhevsky et al., 2012; Kato et al., 2002). Both monocular and stereo vision systems that are capable of tracking and classifying pedestrians and other traffic participants are already on the market and are used in many of the high end ADAS systems in modern cars. However, when focusing on fully autonomous driving further improvements of these systems are needed. The PROUD project had extensive use of cameras (Broggi et al., 2015) and the Bertha project uses cameras both for localization and obstacle detection (Ziegler et al., 2014b). Two main approaches for camera based localization is the lane marking and feature based techniques. Both approaches need the creation of an a priori map in a similar way as the LiDAR based approaches. In the feature based approaches images are recorded and stored in an offline database. By calculating invariant features such as the Scale-Invariant Feature Transform (SIFT) (Lowe, 1999) or Binary Robust Independent Elementary Features (BRIEF) (Calonder et al., 2010) and match them to the images in the database, accurate localization can be obtained (McManus et al., 2014; Lategahn and Stiller, 2014).

Instead of storing features, Schreiber et al. (2013) uses a database of recorded lane markings that are matched to the current visible lane markings and work from Ward and Folkesson (2016) and Lundgren et al. (2014) have tried RADAR based matching for localization. Again a big problem for map based localization is being able to keep a consistent map with changing lightning and weather conditions.

As mentioned before CNNs has revolutionized image classification in recent years and pixel level semantic segmentation now produces impressive results that can distinguish pedestrians, vehicles, vegetation, road surface, sidewalks etc. in an efficient manner (Long et al., 2015). Using this together with dense stereo matching and the stixel-world representation, Schneider et al. (2016) has created a compact and informative 3D representation of the surrounding environment that is suitable for further processing and obstacle map creation.

\section{Scene understanding}

Using the information extracted in the perception and localization layer, the scene understanding layer fuses it into a more compact representation that can be used in decision and 


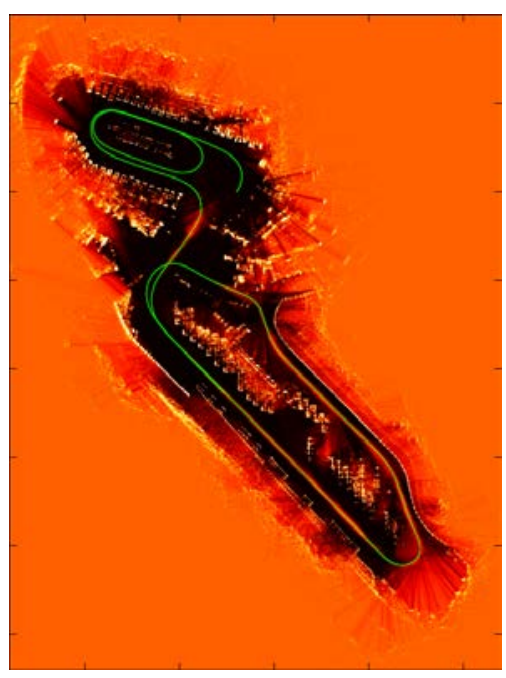

(a)

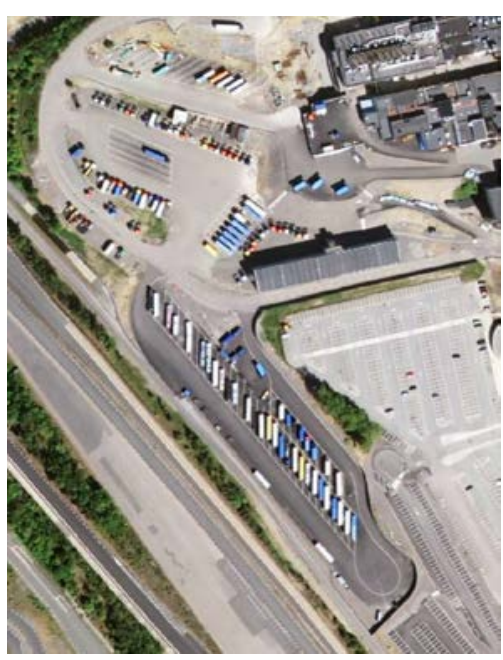

(b)

Figure 1.4: (a): Occupancy grid of the area shown in (b), created using RADAR sensors. Black represents zero or low probability of an obstacle while white represents one or high probability of an obstacle. The orange are unknown areas with 0.5 probability of either an obstacle or free space. The images are taken from Moritz and Pettersson (2014) and is used with courtesy of the authors.

planning layers. Static and dynamic objects are often treated separately and need different handling due to the time dependency of dynamic objects. Prior information of traffic rules and road network structure can be used in combination with models of other vehicles and pedestrians to make predictions of future intentions that need to be considered in decision making.

Depending on the choice of algorithm that will use the information, different representations must be used. A common approach in robotics is to represent static obstacles using occupancy maps where the world is discretized into a two or three-dimensional grid where each grid cell contains a number representing the probability of that cell being occupied or not. Using probabilistic sensor models and Bayesian estimation, Elfes (1989) created occupancy maps that were used for robot navigation in both indoor and outdoor environments. Different sensor modalities are easily handled and fused in the occupancy map by using individual sensor models. The simple representation allows for efficient collision checking and the use of graph search algorithms to create motion plans in complex environments. Grid based approaches have also been successfully applied to autonomous vehicle navigation in several projects e.g. (Broggi et al., 2015; Thrun et al., 2006; Urmson et al., 2008; Montemerlo et al., 2008). As an example, Figure 1.4 shows an occupancy map that was created while driving on a parking lot using RADAR sensor.

Grid based occupancy maps are not suitable for all types of motion planning algorithms. Ziegler et al. (2014a) created polygonal constraints so that they could be incorporated in a continuous, optimization based trajectory planner. However, this approach 


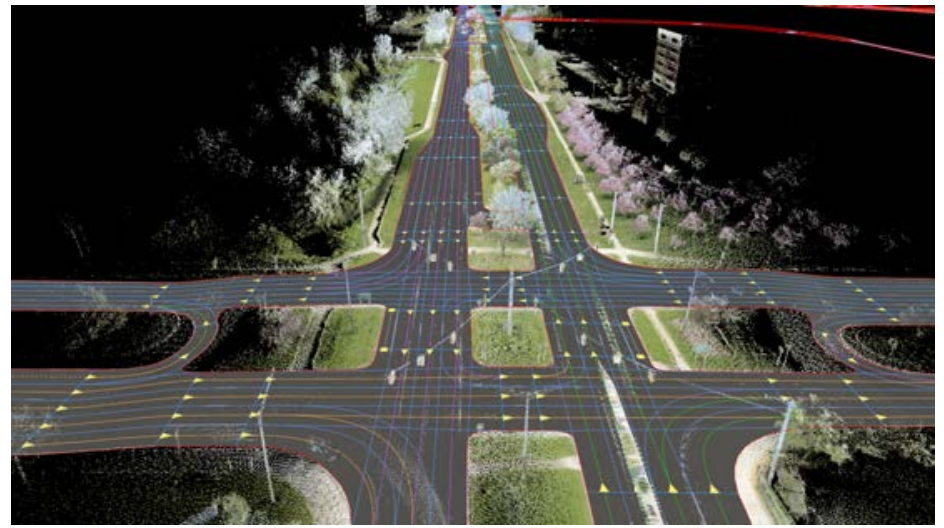

Figure 1.5: A high definition map of an intersection in Michigan complete with $3 D$ point clouds and lane information where arrows represents driving direction and the different lane centers. The image is used with courtesy of HERE.

limits flexibility since convex corridors must be created in order to solve the optimization of the trajectories.

Moving or dynamic obstacles are not handled in a good way by using occupancy maps and need a different approach. Dealing with unpredictable human drivers and pedestrians is a challenging problem and is getting a lot of attention in the autonomous vehicle community. The future trajectories or intentions must be predicted so they can be handled in a safe way while still allowing the autonomous vehicle to progress and not being over conservative. The future trajectories of other vehicles are often predicted using the assumption of constant velocity (Ziegler et al., 2014b; Werling et al., 2008; Ferguson et al., 2008). This assumption is often valid in simpler situations but with increasing complexity of traffic situations, intentions such as lane changes, yielding at intersection or turning needs to be predicted (Liebner et al., 2013; Schlechtriemen et al., 2015; Ward and Folkesson, 2015). Further complicating the prediction is the dependency on our own actions. Depending on what decision the ego vehicle will take, the action of other vehicles might change. To deal with this social interaction some interesting approaches have been presented (Wei et al., 2013; Cunningham et al., 2015; Eggert et al., 2015) and is also studied in Paper D of this thesis.

\section{Decision making and planning}

Decision and planning are often done in a hierarchical structure. On the highest level a mission planning module receives a destination from the passenger or in the mining case a site operator. For an autonomous vehicle on public roads the mission planning calculates, in a similar way to every day navigation aids, a high level plan that could be the shortest, fastest or most fuel efficient path given the road network. The plan contains information of which streets to take and preferred lanes to keep. Detailed information about the road network is needed to construct a plan and an example of a high definition map used for autonomous driving is shown in Figure 1.5. For a mining application, goals 
are received from a site operator with task such as where to go to be loaded and where to dump material and then again a high level plan is calculated according to some metric of cost. Once the high level plan has been established there are usually two different type of areas that are encountered, namely structured areas with well-defined rules and paths where to drive, such as streets and highways and then unstructured areas such as parking lots or a mining floor in an open pit mine. These two situations are usually handled separately by different algorithms. In structured areas planning involves behavior selection such as if it is beneficial to overtake a vehicle or remain in a vehicle following behavior. Once the behavior has been decided, it is up to the motion planning algorithm to generate a trajectory that implements the behavior while abiding to obstacle constraints and maintaining dynamically feasible motions for the vehicle. A driving reference along the center of the road is often given and planning only involves maneuvers deviating around the reference path. In unstructured areas, very general environments are handled and the main problem consists of finding a motion plan, around sometimes, very tight obstacle constraints, while exploiting the full kinematic capabilities of the vehicle.

Motion planning in unstructured areas is a well explored problem and many solutions still build on graph search algorithms, such as $A^{*}$, from early work by Hart et al. (1968). These techniques have been further developed and is the work horse for many planning algorithms for unstructured areas deployed in real systems (Urmson et al., 2008; Montemerlo et al., 2008). More recent efforts by LaValle (1998), with randomized sampling based algorithms have also been used and deployed for both structured and unstructured planning in work by Kuwata et al. (2009).

For structured area trajectory generation prior information about the center of the road is often exploited. Urmson et al. (2008) sampled a set of points down the road from the vehicle with different offset around the reference. Then a vehicle model was used in an optimization step where optimal trajectories from the current vehicle state to the sampled points were calculated and then checked for feasibility and finally scored in a cost function. Montemerlo et al. (2008) used a similar strategy with trajectory roll-outs. Werling et al. (2011) further developed these ideas and transformed the problem into the Frenet frame around the path where a vast number of candidate trajectories with different offsets can be created extremely efficiently and then be combined with different speed profiles. All generated trajectories are checked both in space and time against the static obstacles and the predicted movements of all dynamic obstacles before the most suitable trajectory is selected and sent to low level controllers for execution. These steps are then repeated, often several times per second so newly received sensor information can be accounted for.

\section{Trajectory control}

Once a feasible trajectory has been determined in the motion planning layer, low-level feedback controllers stabilize the car around the given trajectory. Controllers are often running at a high update frequency typically between $50-200 \mathrm{~Hz}$ and depending on speed, dynamics of the vehicle and the complexity of the trajectories to follow, different approaches have been used. Trajectory tracking can be divided into two control problems, the longitudinal and lateral. Longitudinal stabilization involves the problem of velocity control to make sure the vehicle progresses along the path and arrives at the correct 
time instances along the path while lateral control steers the vehicle to minimize the lateral deviation from the specified path. Coulter (1992) developed the simple yet effective pure-pursuit lateral path following controller that was used for the NavLab platform and many teams in the DARPA Challenges (Thrun et al., 2006; Kuwata et al., 2009). The pure-pursuit controller is based on purely geometric relations and vehicle dynamics are not modeled which gives an easy implementation but can give tracking problems or even instability at higher speeds. Since the pure-pursuit controller only concerns lateral stabilization other controllers are needed for the longitudinal stabilization. In simpler settings, PI-controllers are often enough, but if exact longitudinal control is needed at all times drive train dynamics need to be considered in the design (Attia et al., 2014).

With advances in optimization tools and methods, real-time performance of Model Predictive Controllers (MPC) have become possible and gives the possibility to incorporate vehicle constraints and delays in the control design (Falcone et al., 2008; Borrelli et al., 2005; Lima et al., 2016). This has allowed for stable operation in normal operating conditions at higher speeds and similar techniques have even been investigated by Beal and Gerdes (2013) for control at the limits of the handling capabilities.

\subsection{Thesis outline}

This thesis is divided into two parts, with edited versions of published papers in the second part. The main work in this thesis is done in the motion planning and decision layer of an autonomous system but it also touches some aspects of scene understanding and particularly the prediction of other vehicles. Part one starts with Chapter 2 and introduces the standard motion planning algorithms and gives the necessary background needed to read the papers and understand the different applications presented in part two. Chapter 4 ends part one by summarizing the conclusions and discusses directions for future work.

Part two contains edited versions of four published papers and a summary of each paper is given below:

\section{Paper A: Sampling Recovery for Closed Loop Rapidly Expanding Random Tree using Brake Profile Regeneration}

Niclas Evestedt, Daniel Axehill, Marco Trincavelli, and Fredrik Gustafsson. Sampling Recovery for Closed Loop Rapidly Expanding Random Tree using Brake Profile Regeneration. In 2015 IEEE Intelligent Vehicles Symposium (IV), pages 101-106. IEEE, 2015.

Summary: This paper presents a simple yet effective extension to the sampling step in the CL-RRT framework. Sampling in a completely random manner when dealing with real-time implementations of sampling based algorithms on dynamic platforms can give problems in critical situations when a safe solution needs to be found fast. To mitigate the risk of not finding a feasible path when operating in an unknown unstructured area, we propose the introduction of a deterministic break profile calculation in the tree construction phase that uses information from the forward simulation to calculate an acceleration profile that, if possible, stops the vehicle before reaching the obstacle when a new branch has been found unfeasible. The approach is tested with a real implementation on a heavy 
duty Scania G480 mining truck in a simple constructed scenario.

Background and Contribution: Parts of the original CL-RRT algorithm developed by Kuwata et al. (2009) was implemented by the author as part of a demonstration within the iQMatic project. The algorithm was integrated into the system on one of the project's test vehicles and has been used successfully during several demonstration events. During this work the author of this thesis came up with the idea for the paper, did the necessary implementation and did all of the writing. Data collection was done in collaboration with Marco Trincavelli at Scania. Assoc. Prof. Daniel Axehill and Prof. Fredrik Gustafsson acted as supervisors and reviewed the manuscript.

\section{Paper B: Path tracking and stabilization for a reversing general 2-trailer config- uration using a cascaded control approach}

Niclas Evestedt, Oskar Ljungqvist, and Daniel Axehill. Path tracking and stabilization for a reversing general 2-trailer configuration using a cascaded control approach. In 2016 IEEE Intelligent Vehicles Symposium (IV), pages 1156 - 1161. IEEE, 2016b.

Summary: In this paper a cascaded control approach for stabilization and path tracking for a general 2-trailer configuration is presented. The nonlinear equations for the 2 trailer configuration are linearized around stationary equilibrium configurations and then stabilized using linear quadratic control (LQ). A pure-pursuit path tracking controller is then designed on top in a cascaded structure to allow for path following of piecewise linear reference paths. An easy to use driver aid is then developed that can be used to manually plan complex maneuvers that the platform can execute. The system is then tested in both simulation and on a small scale test platform.

Background and Contribution: The original concept for the project on reversing trailers was initiated from discussions between the author of this thesis and Assoc. Prof. Daniel Axehill. This resulted in a proposal for a master's thesis project that was assigned to Oskar Ljungqvist and a tight cooperation was initiated. Low level controller design, based on work by Altafini et al. (2002), was developed by Oskar Ljungqvist and the main ideas for the user interface were designed and implemented by the author of this thesis. Path tracking, experimental platform development and data collection was accomplished jointly between the author and Oskar and the majority of the writing was done by the author of this thesis.

\section{Paper C: Motion planning for a reversing general 2-trailer configuration using Closed-Loop RRT}

Niclas Evestedt, Oskar Ljungqvist, and Daniel Axehill. Motion planning for a reversing general 2-trailer configuration using Closed-Loop RRT. In 2016 IEEE/RSJ International Conference on Intelligent Robots and Systems (IROS), pages 3690 - 3697. IEEE, 2016a.

Summary: This paper presents a motion planning framework based on CL-RRT for the general 2-trailer configuration. The stabilizing controllers developed in Paper B are 
used for forward simulations within the CL-RRT framework developed in Paper A. The framework is then evaluated in a series of simulation experiments in different kinds of environments and is shown to have a high success rate in finding complex motion plans in complex and constrained environments.

Background and Contribution: The idea of using stabilized models of the general 2-trailer configurations within the CL-RRT framework was initiated from discussions between the author of this thesis and Assoc. Prof. Daniel Axehill. The concept was then tested as part of Oskar Ljunqvist's master's thesis and further developed for the results in this paper. Development and implementation of the core parts of the CL-RRT framework was done by the author of this thesis and integration of the model into the CL-RRT framework, experimental platform development and data collection was accomplished jointly between me and Oskar. The majority of the writing was done by the author of this thesis and Assoc. Prof. Daniel Axehill acted as supervisor and reviewed the manuscript.

\title{
Paper D: Interaction aware trajectory planning for merge scenarios in congested traffic situations
}

\author{
Niclas Evestedt, Erik Ward, John Folkesson, and Daniel Axehill. Interaction \\ aware trajectory planning for merge scenarios in congested traffic situations. \\ In 2016 IEEE Intelligent Transportation Systems Conference (ITSC), pages \\ 465 - 472. IEEE, 2016c.
}

Summary: Paper A-C have considered the motion planning problem in unstructured areas while this paper looks into problems concerning motion planning in structured areas with, sometimes, heavy traffic. Previous methods for planning in urban scenarios usually use conservative constant velocity models for other traffic participants that ignore the social interaction between the ego vehicle and other vehicles. In this paper we show, by simulations, that these models are too conservative when dealing with heavy traffic and instead we introduce a prediction step, based on the Intelligent Driver Model (IDM), where the social interaction can be predicted in a simulation step before an action is chosen. Dealing with heavy duty vehicles this problem is even greater and truck drivers often have to force its way out in traffic.

We propose a sampling based method where a vast amount of candidate trajectories is generated and then for each trajectory the traffic scene is simulated and predicted in response to our chosen trajectory. Each trajectory is then scored according to cost functions incorporating both progress, comfort and risk before the most suitable trajectory for the ego vehicle is sent for execution. The framework is then implemented and tested in simulation with real time performance at $10 \mathrm{~Hz}$.

Background and Contribution: The collaboration with Erik Ward was initiated through the iQMatic project where both are involved in the development of the decision making framework. The main idea for the paper came out from many discussions between the author and Erik about how to handle and predict other vehicles within a motion planning framework. Coding and implementation of the framework and data collection was accomplished jointly between the author and Erik and the majority of the writing 
was done by the author of this thesis. Assoc. Prof. Daniel Axehill and Asst. Prof. John Folkesson acted as supervisors and reviewed the manuscript.

\subsection{Contributions}

The author is particularly proud to present the following scientific contributions in this thesis.

- A sampling recovery step for use in real-time RRT applications. This was presented in Paper A.

- The incorporation of the cascaded control framework, presented in Paper B, into the CL-RRT framework presented in Paper C. To the authors knowledge this was the first planning framework considering the general 2-trailer configuration with off-hitch.

- A real-time capable method for incorporating the social interaction between ego vehicle and other traffic participants in merging scenarios. This was presented in Paper D. 


\section{2}

\section{Classical motion planning}

This chapter introduces the robot motion planning problem in its original formulation and presents some of the most successful algorithms used to solve it. The original motion planning formulation, often called The Piano Mover's Problem, consist of moving a geometrically complicated object, such as a piano, through a complicated environment, such as a house, without hitting any of the obstacles inside the house. In robotics, instead of moving a piano, a robot is considered and the problem is to get the robot to automatically reason about the environment and develop a plan of how to move through the environment without hitting obstacles.

The original formulation only considers pure geometrical reasoning and holonomic platforms, i.e. objects are allowed to move and rotate in any direction in contrast to non-holonomic platforms that are constrained and cannot move in all directions. Nonholonomic platforms increase the complexity of the motion planning problem and unfortunately they are common in real applications, e.g. cars, airplanes, manipulators etc., but practical methods for some of these systems exist and important insight is gained by first studying the classical motion planning formulation.

Dynamic and changing environments, path optimality, complex dynamic models, high dimensional systems, moving obstacles etc. further complicate the problem but are neglected in this chapter. However, some of these aspects are discussed later in Chapter 3.

\subsection{Configuration space}

This section will give a brief overview of concepts and algorithms concerning motion planning. For a comprehensive study and more details of algorithms the reader is referred to one of the many text books published on the subject (Choset et al., 2005; LaValle, 2006; Latombe, 2012).

Informally the $2 \mathrm{D}$ motion planning problem can be formulated as, let $\mathcal{W}=\mathbb{R}^{2}$ denote 

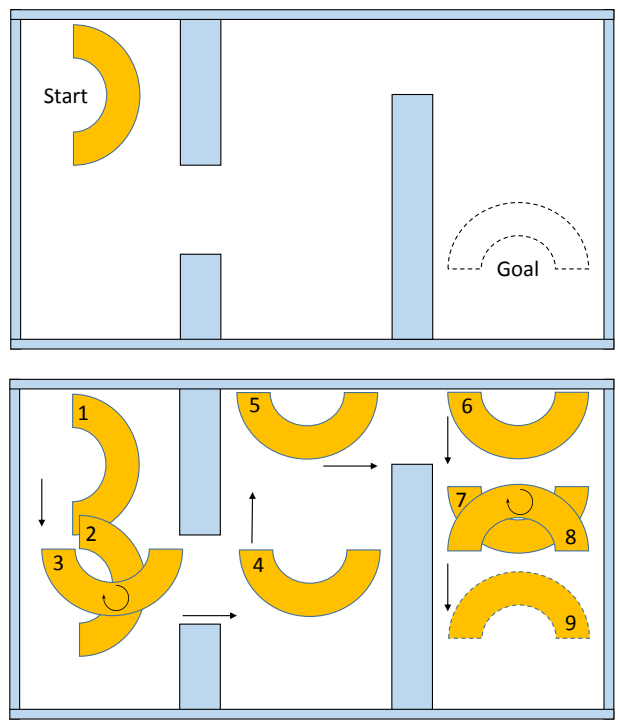

Figure 2.1: Example of a geometrical planning problem. The task is to move the semi-circular object from a start position to an end position by a sequence of gradual movements represented by the numbering on each gradual movement.

a $2 \mathrm{D}$ world with the polygonal obstacle region $\mathcal{O} \subset \mathcal{W}$ and containing a rigid robot body with polygonal boundary. Given an initial placement and a goal placement of the robot, compute a sequence of gradual movements that moves the robot from the initial placement to the goal placement without hitting any obstacles. Figure 2.1 shows such a sequence for a semi-circular shaped robot moving in a constrained environment.

The motion planning problem is described in the world $\mathcal{W}$, but the problem really lives in another space called the configuration space or the $\mathcal{C}$-space. Defining $q=(x, y, \theta)$ to be the configuration or pose of a robot in the $2 \mathrm{D}$ world, where $(x, y)$ represents the position of the origin of the robot in the $x, y$-plane and $\theta$ represents the rotation around a fixed axis, the configuration space $\mathcal{C}$, is the space of all possible robot configurations. The configuration space is an important concept in Lagrangian mechanics and was introduced in motion planning by Udupa (1977) and Lozano-Perez (1983). Defining the motion planning problem in the $\mathcal{C}$-space, transforms the problem to become a search in a space of transformations and allows for the development of algorithms that are independent of the shape or kinematics of the robot. In the $2 \mathrm{D}$ world the robot position is represented in $\mathbb{R}^{2}$ and the rotation in the group of all rotations around the origin, $S O(2)$. $S O(2)$ denotes a circle where 0 and $2 \pi$ is "fused" together to account for the "wrap around" at $2 \pi$. To avoid unnecessary redundant exploration by the introduction of artificial barriers it is important to correctly account for the "wrap around" when implementing motion planning algorithms.

The $\mathcal{C}$-space for the $2 \mathrm{D}$ world with rotations now becomes $\mathcal{C}=\mathbb{R}^{2} \times S O(2)$ which is of dimension 3 and the $\mathcal{C}$-space without rotations becomes $\mathcal{C}=\mathbb{R}^{2}$. The concept extends 

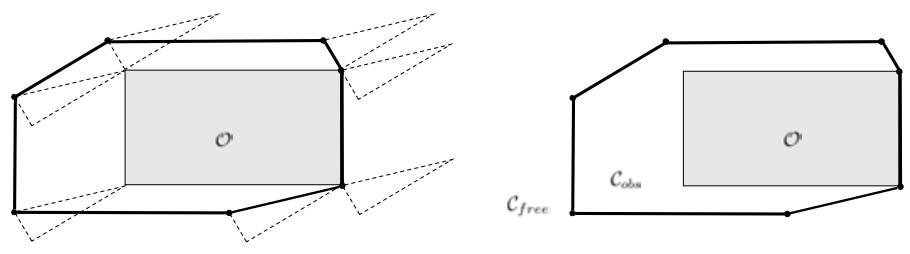

Figure 2.2: A simple example from LaValle (2006) where the origin of a triangular shaped robot $\mathcal{A}$, is traced and slided along the obstacle region $\mathcal{O}$, to create the parts $\mathcal{C}_{\text {obs }}$ of the $\mathcal{C}$-space that are in collision.

easily to higher dimensional robots and worlds but the shape of the $\mathcal{C}$-space becomes much more complex.

Let $\mathcal{A}(q) \subset \mathcal{W}$ be the closed set of all robot points that occupy the world $\mathcal{W}$, when the robot is in configuration $q$. A configuration $q \in \mathcal{C}$ is said to be in the free space if the robot $\mathcal{A}(q)$, is not in contact with any point in the obstacle set $\mathcal{O}$. The free space $\mathcal{C}_{\text {free }}$, which is the set of all configurations that are not in collision with $\mathcal{O}$, can now be defined as

$$
\mathcal{C}_{\text {free }}=\{q \in \mathcal{C} \mid \mathcal{A}(q) \cap \mathcal{O}=\emptyset\}
$$

which gives the obstacle region

$$
\mathcal{C}_{\text {obs }}=\mathcal{C} \backslash \mathcal{C}_{\text {free }}
$$

By sliding the robot along the edge of the obstacle region and tracing the position of the robot origin, $\mathcal{C}_{\text {obs }}$ can be obtained. An example for a triangular robot in the $2 \mathrm{D}$ world without rotations is illustrated in Figure 2.2 and the case with rotations for a curved robot inside a constrained space with a narrow channel between two small areas is illustrated in Figure 2.3. Already for this simple example the shape of $\mathcal{C}_{\text {free }}$ is quite complicated. Once $\mathcal{C}_{\text {free }}$ is defined the motion planning problem is reduced to finding a path for a point moving through $\mathcal{C}_{\text {free }}$. More formally, given a robot description $\mathcal{A}$, an obstacle region $\mathcal{O}$, a configuration space $\mathcal{C}$, an initial configuration $q_{\text {init }} \in \mathcal{C}_{\text {free }}$ and a goal configuration $q_{\text {goal }} \in \mathcal{C}_{\text {free }}$ the motion planning problem consists of computing a continuous path $\tau$ : $[0,1] \rightarrow \mathcal{C}_{\text {free }}$ with $\tau(0)=q_{\text {init }}$ and $\tau(1)=q_{\text {goal }}$. 

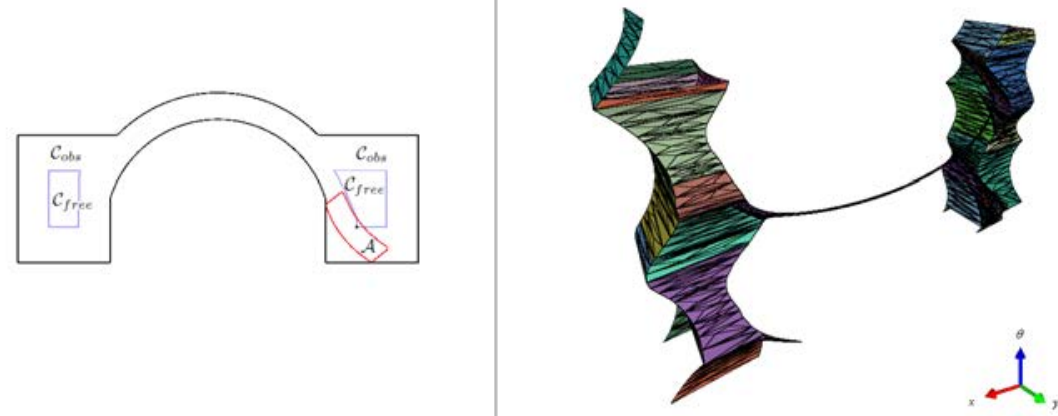

Figure 2.3: Illustration of the 3-dimensional $\mathcal{C}$-space for the robot $\mathcal{A}$ when rotations are introduced. The robot is slided along the obstacle region in the same way as the $2 D$ example but now for every possible orientation $\theta$ to create the complex shape shown on the right side.

\subsection{Popular approaches}

There are three main schools with different approaches of how to handle this problem, combinatorial methods, sampling-based methods and artificial potential field methods. Before looking closer at these algorithms, some properties of motion planning algorithms need to be defined. A motion planning algorithm is said to be:

Complete if the planner always finds a solution in finite time if one exists or terminates and reports failure if one does not exist.

Resolution complete if the planner always finds a solution, within a specified resolution, in finite time if one exists or terminates and reports failure if one does not exist.

Probabilistically complete if a solution exists, the probability of finding one goes to one as the number of iterations tends to infinity.

Completeness is an attractive property for any motion planning algorithm. However, approximations have to be made for most practical problems, and weaker completeness guarantees have to be accepted. The $\mathcal{C}$-space is a continuous space and needs to be discretized in order to perform computations.

Combinatorial methods were developed during the 1980s and offer an efficient, exact and complete solution to many special classes of problems. However, they are usually not of practical interest and quickly become intractable when the dimension of the $\mathcal{C}$-space increases. These algorithms need an algebraic, often a polygonal, representation of the obstacle region $\mathcal{O}$. They work by constructing a graph in $\mathcal{C}_{\text {free }}$ that completely captures the structure of the free space, often called a roadmap, where each vertex is a configuration $q \in \mathcal{C}_{\text {free }}$ and each edge is a collision free path through $\mathcal{C}_{\text {free }}$. Once the roadmap is 
constructed it can be used for multiple queries and searched by graph search methods to find paths from different initial and goal configurations. Visibility graphs, Voronoi diagrams and Exact cell decomposition are some common methods for combinatorial planning and interested readers are refereed to textbooks by LaValle (2006), De Berg et al. (2008) or Latombe (2012).

Artificial potential field methods is another approach initially developed for online reactive motion planning but it has also been found useful for global path planning problems. The idea is to create a potential field $\mathcal{U}$, where the robot moves under the action of a force generated from the field. A combination of an attractive potential towards the goal and a repulsive potential from obstacles in $\mathcal{C}_{\text {obs }}$, creates a force that pushes the robot towards the goal but at the same time creates a repulsive force that avoids obstacles. Motion planning in this field is essentially using gradient decent and in every step the robot executes a motion in the direction of the negative gradient, $-\nabla \mathcal{U}(q)$. Even though a unique global minimum exists the problem with such methods is the many local minima where the robot can get trapped. Workarounds to create fields without local minima and the local use of other methods to backtrack and escape have been tried and again more information can be found in Latombe (2012) or Barraquand and Latombe (1990, 1991).

Sampling based methods are by far the most applied methods for real world problems and have proven to be useful for a broad range of problems. These are also the family of methods that has been used to solve many of the problems presented in this thesis and therefore will be given a more thorough introduction.

Instead of fully characterizing $\mathcal{C}_{\text {free }}$ such as the previously discussed methods, sampling based methods have no prior information about the configuration space and instead use a collision detection module to probe configurations in $\mathcal{C}$-space to see if a given configuration is in collision or not. The collision detection module is seen as a "black box" and can be designed independent of the algorithm. In this way the algorithms become independent of the geometrical configuration or kinematics of the robot, however, efficient collision detection modules need to be developed in order to reach acceptable performance. The algorithms use the collision detection module to incrementally probe and search the $\mathcal{C}$-space to reveal more and more parts which are contained in $\mathcal{C}_{\text {free }}$. It might be difficult to directly realize what is gained by this approach but by intelligently choosing which parts to probe, large spaces of unnecessary exploration can be avoided and a solution can be found without fully revealing the complete structure of the $\mathcal{C}$-space. Unfortunately, weaker completeness guarantees such as resolution completeness or probabilistic completeness are offered by these methods but this is seldom an issue and is thus acceptable for most practical problems. Sampling based methods store the explored parts of $\mathcal{C}_{\text {free }}$ in a graph structure. Two main approaches of how to build the graph exist, discrete deterministic methods that often use standard graph search algorithms to find solutions and the more recent probabilistic sampling based methods that explore the connectivity of configurations in a randomized manner.

\subsubsection{Discrete methods}

Discrete methods often discretizise $\mathcal{C}_{\text {free }}$ using graph data structures, so a short introduction of graphs and discrete state spaces will be given.

A graph is a set containing objects where some pairs of objects have a relationship. 

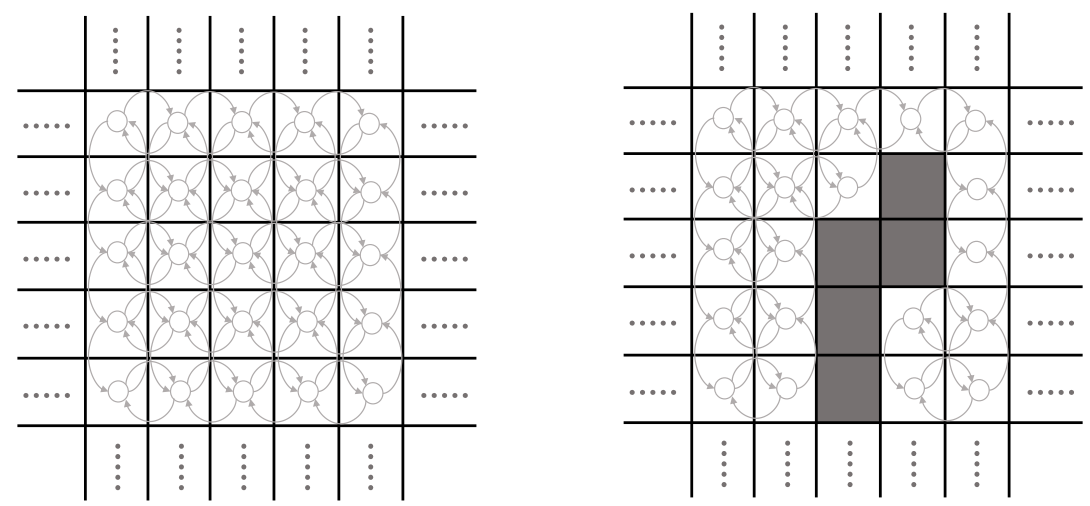

Figure 2.4: Example of the state transition graph for a simple robot that can move up, down, left and right on a discretized $2 D$ grid. An arrow represents an edge with a possible action $u$. If some grid cells are occupied, as illustrated by the gray areas to the right, the corresponding edges are removed and only movements in the free space are allowed.

The objects are called vertices or nodes and each pair of related objects are called an edge. Edges can either be undirected or directed e.g. two houses, A and B, that are connected with a one-way street would generate a directed edge where the vertices or nodes would correspond to the two houses and the edge to the relationship that it is possible to move from A to B. If the street would have two lanes the edge would be undirected since it is possible to move from both $\mathrm{A}$ to $\mathrm{B}$ and from $\mathrm{B}$ to $\mathrm{A}$. A graph, $\mathcal{G}$ is now defined as an ordered pair $\mathcal{G}=(\mathcal{V}, \mathcal{E})$ where

- $\mathcal{V}$ is a set of vertices or nodes

- $\mathcal{E}$ is a set of unordered or ordered pairs of vertices that represent undirected or directed edges, respectively.

To make this definition useful for planning the discrete state transition graph is defined. Each possible situation in the world is referred to as a state $x$, and the set of all possible states is called the state space $\mathcal{X}$. This set needs to be a finite or countable infinite set of states in order for discrete methods to work, but in general it can be continuous. The state can be manipulated through an action or input $u$, that when applied to a state $x$, produces a new state $x^{\prime}$ that is specified by a state transition function as

$$
x^{\prime}=f(x, u)
$$

The set of all actions or inputs that can be applied to a state is contained in the input or action space $\mathcal{U}$. The set of vertices for the state transition graph now corresponds to the 
state space $\mathcal{X}$ and an edge between $x$ and $x^{\prime}$ only exists if there is an action $u \in \mathcal{U}$, that produces a valid transition from $x$ to $x^{\prime}$. The state transition graph for a robot that can move up, down, left and right in a state space represented by an $(x, y)$-grid is shown in Figure 2.4. An arrow from one state to another represents a possible transition and for this example the state $x$, is represented by all integer pairs $x=\left(x_{i}, y_{i}\right) \in \mathbb{Z}^{2}$ and represents a coordinate on the grid. The state transition function is $f(x, u)=x+u$ with possible inputs $u \in \mathcal{U}=\{(0,1),(0,-1),(-1,0),(1,0)\}$ where each pair represent the movements up, down, left and right, respectively. Starting in state $x=(1,2)$ and applying the state transition equation with an upward movement will result in a new state $x^{\prime}=f(x, u)=$ $x+u=(1,2)+(0,1)=(1,3)$. Introducing regions on the grid that represent obstacles and are illegal to occupy will remove the corresponding vertices and edges in the state transition graph and constrain the movement of the robot to remain in valid areas.

Assuming a point like robot, a search in $\mathcal{C}_{\text {free }}$ corresponds to traversing this state transition graph using a collision detection method that would simply be to check if the coordinates $\left(x_{i}, y_{i}\right)$, for the resulting or expanded state $x^{\prime}$ is marked as an obstacle or not. Starting at a given initial state $x_{\text {init }}$, and a desired goal state $x_{\text {goal }}$ the discrete motion planning problem is summarized by LaValle (2006) as

1. A non-empty state space $\mathcal{X}$, which is a finite or countable finite set of states.

2. For each state $x \in \mathcal{X}$, there is a finite input or action space $\mathcal{U}(x)$.

3. A state transition function $f$ that produces a new state $x^{\prime}=f(x, u) \in \mathcal{X}$ for every $x \in \mathcal{X}$ and $u \in \mathcal{U}(x)$.

4. An initial state $x_{\text {init }} \in \mathcal{X}$.

5. A goal set $\mathcal{X}_{\text {goal }} \subset \mathcal{X}$.

The task of the planning algorithm is then to find a finite sequence of inputs that when applied transforms the initial state $x_{\text {init }}$ to some state in $\mathcal{X}_{\text {goal }}$. To find this sequence, discrete sampling based search methods start from the initial state (some algorithms can start from the goal and search backwards) and apply all valid inputs $u \in \mathcal{U}(x)$ at that state. The resulting states $x^{\prime}$ for each of the inputs are then evaluated using the collision detection method to determine if they are in a valid configuration or not. If the resulting states are valid, all inputs are again applied to the newly expanded states and the same steps are repeated until the goal is found or all possible states have been visited and the algorithm reports failure to find a solution.

This expansion of states then corresponds to performing a basic graph search on the state transition graph with the only difference that the full graph is not known in advance and needs to be explored by applying actions and the collision detection module. A wide range of different algorithms for graph search have been developed in the computer science community and basically the only difference between algorithms is in the ordering they choose to expand states. However, this ordering is crucial for different properties of the algorithm and Cormen et al. (2009) gives a great summary of different graph search algorithms. $\mathrm{A}^{*}$ is the main workhorse for solving many of the discrete motion planning problems and many related algorithms have been derived from its working principles. Before presenting the $A^{*}$ algorithm, Dijkstra's algorithm introduced by Dijkstra (1959), from which $\mathrm{A}^{*}$ is easily derived, will be presented. 


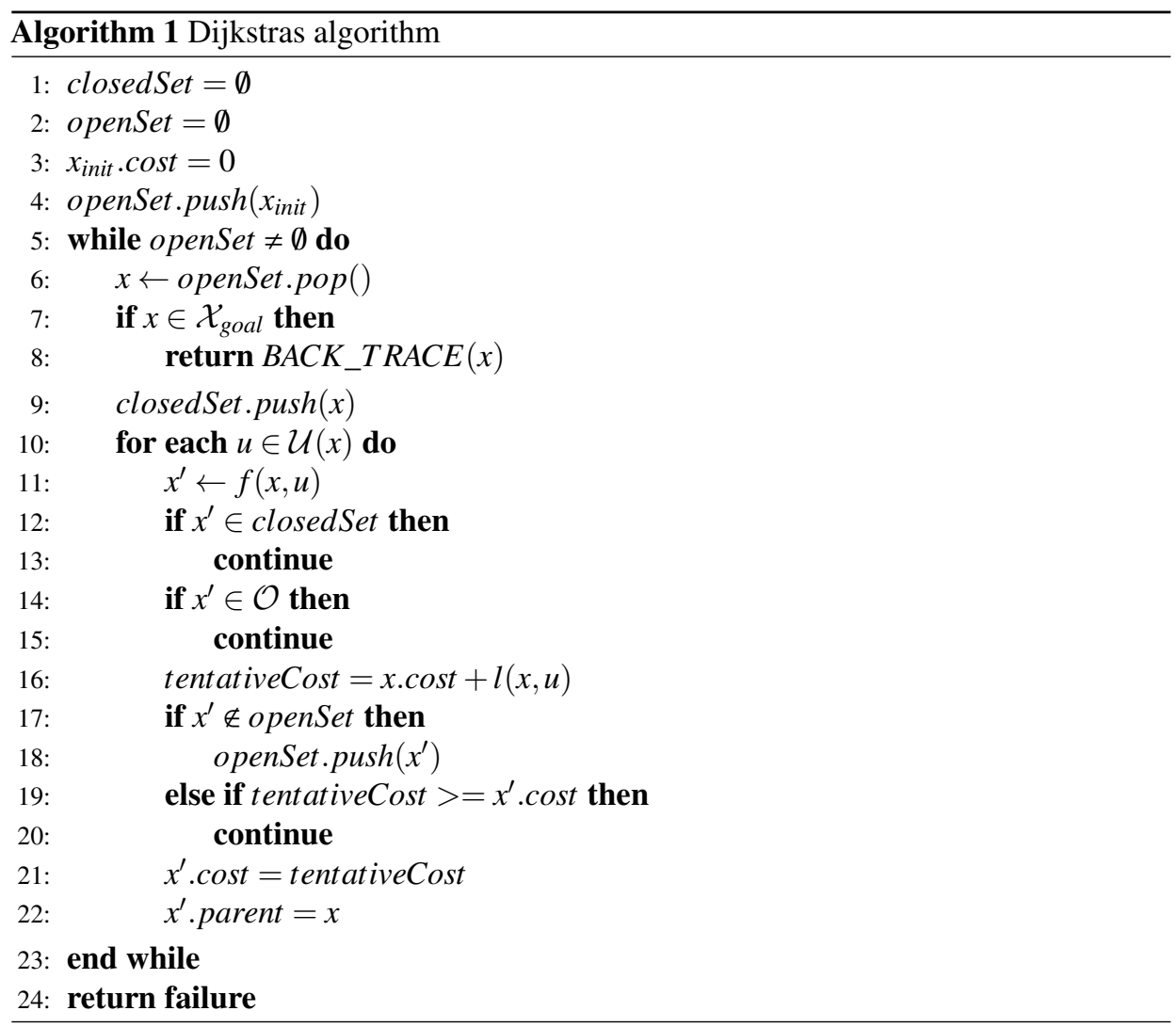

\section{Dijkstras algorithm}

Dijkstras algorithm is used for finding the shortest path between nodes in a graph, not only will it find a feasible sequence of actions it will also find the sequence with the lowest overall cost to get from $x_{\text {init }}$ to $x_{\text {goal }}$ and is said to be optimal. Optimality of a sequence in this sense is often the sequence that gives the resulting shortest travel distance but it could be extended to a criterion incorporating e.g. fuel consumption, time or sometimes even the most scenic path.

Associating a non-negative cost $l(x, u)$ to every action $u \in \mathcal{U}(x)$ gives every edge in the graph a cost for traversing that edge. The cost of the complete task is then the sum of all edge costs when moving from the start node to the goal node. The algorithm maintains two data structures to keep track of which nodes that have been visited and which node that are the next candidate for expansion, namely the closed set $C$, and a priority queue, called the open set $O$, respectively. The nodes in the priority queue are sorted according to the lowest cost-to-come given by $g(x)$, that is the total cost of traveling from the start node to the current node. In this way the next node in the queue will be the one with the so far lowest cost-to-come. The algorithm begins by adding the start state $x_{\text {init }}$ to the open set. It then enters a loop where the first element in the open set is selected and moved to the closed set before each possible action $u \in \mathcal{U}(x)$ is applied and the resulting states 
$x^{\prime}=f(x, u)$ are calculated. If $x^{\prime}$ is a new node that has not been visited before i.e. $x^{\prime} \notin C$ it is checked for feasibility using a collision detection function. If $x^{\prime}$ is a feasible state it is checked to see if it is already in $O$, if not, $x^{\prime}$ is added to $O$ with its associated cost-to-come, calculated by adding the cost to reach the parent state $x$ and the cost of the applied action to reach $x^{\prime}$ from $x$. If $x^{\prime}$ is in $O$ already, the path cost already stored in $O$ is checked against $g\left(x^{\prime}\right)$ to see if the current path is a better path to reach $x^{\prime}$. If the current path is better, the cost of the state is updated and reinserted in $O$. These steps are repeated until the goal state is selected from $O$ and the solution is found or there are no more elements in $O$ and the algorithm reports failure to find a solution. By storing a pointer to the parent node the path to reach $x_{\text {goal }}$ can be backtracked by following the pointer until the start node is reached. The complete algorithm is summarized in Algorithm 1.

To be convinced that this algorithm always finds an optimal path it is important to understand the effect of the cost update at row 19 in Algorithm 1. When an application of an action reveals that the same state is reached again and is already stored in $O$, the cost to reach the state is updated if it has a lower cost than the previously found cost. In this way the costs for nodes in $O$ are always guaranteed to be the lowest cost found so far. Now if a node $x_{\text {current }}$ is selected for expansion it is known that $x_{\text {current }}$ has a lower cost than all other nodes in $O$ since it is sorted according to the cost-to-come and therefore it is impossible to find a new path with a lower cost that leads to $x_{\text {current }}$. If $x_{\text {goal }}$ has arrived to the top of the queue and is selected it is therefore guaranteed that it has been reached by the lowest cost path. A formal proof of optimality based on induction is given by Cormen et al. (2009). The time complexity for Dijkstra's algorithm is highly dependent on implementation details and especially the implementation of the priority queue. The original implementation had a time complexity of $O\left(|\mathcal{V}|^{2}\right)$ where $|\mathcal{V}|$ is the number of nodes and $O$ represents the big $\mathrm{O}$ notation for limiting behavior. By using a Fibonacci-heap implementation of the priority queue the time complexity can be reduced to $O(|\mathcal{V}| \log (|\mathcal{V}|)+|\mathcal{E}|)$ where $|\mathcal{E}|$ is the number of edges in the graph (Cormen et al., 2009). Further improvements can be gained by using prior information about the problem to guide the search to interesting regions and this is the idea behind informed search methods such as $\mathrm{A}^{*}$.

\section{$A^{*}$ algorithm}

The A* algorithm was presented by Hart et al. (1968) as an extension to Dijkstra's algorithm. It tries to reduce the total number of explored states by incorporating a heuristic estimate of the cost from a state $x$ to the goal node $x_{\text {goal }}$, called the cost-to-go, and use this information to select the node that shows most promise to reach the goal quickly.

$A^{*}$ is following the exact same steps as Dijkstra's algorithm with the only difference that the priority queue for the open set $O$ is sorted in a different way. Instead of only looking at the cost-to-come, $\mathrm{A}^{*}$ also use a heuristic function $h(x)$ that returns an underestimate of the true cost-to-go and sorts the priority queue according to $f(x)=g(x)+h(x)$ where $g(x)$ is the cost-to-come for node $x$ and $f(x)$ is an estimate of the total cost of a path from start to goal. If $h(x)=h^{*}(x)$ where * indicates the true cost-to-go of the optimal path, then $\mathrm{A}^{*}$ would expand only nodes on the optimal path and find it without any unnecessary exploration. However, $h^{*}(x)$ is never available in advance as knowing it would correspond to solving the initial problem. Fortunately, underestimates of $h^{*}(x)$ is possible to derive 


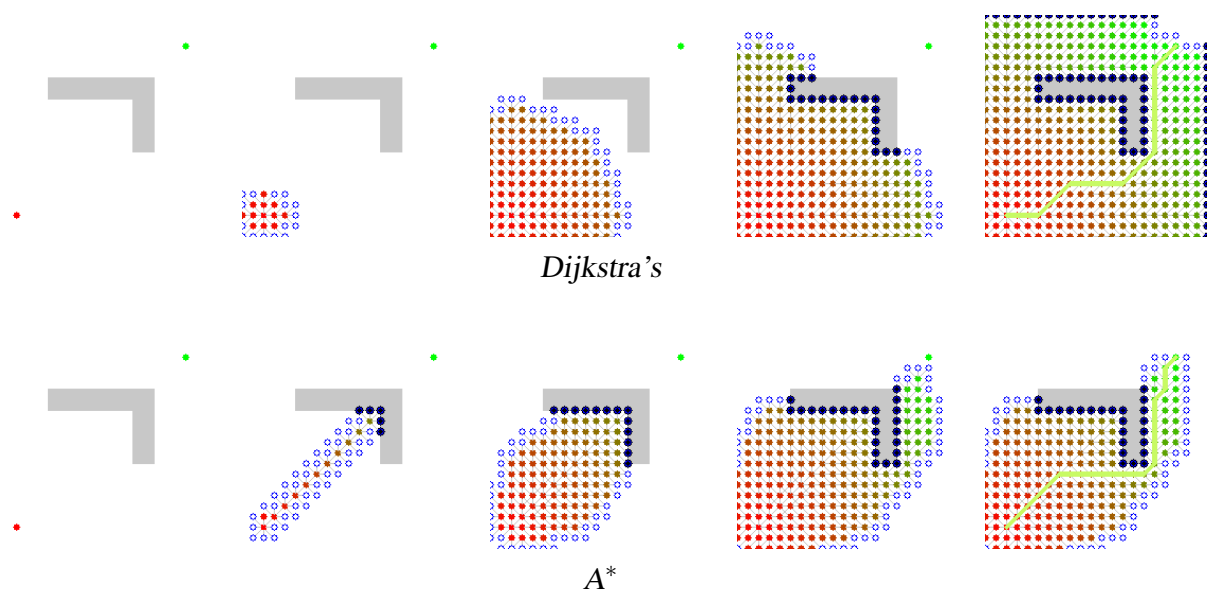

Figure 2.5: Comparison between the number of expanded nodes for Dijkstra's and $A^{*}$ algorithms when solving an identical problem. Dijkstra's algorithm has a circular expanding search pattern that explores almost all nodes before reaching the goal in contrast to $A^{*}$ that guided by the Euclidean distance heuristic shoots straight for the goal and needs to explore far less nodes. Light blue nodes represent the nodes in the open set and the other nodes are color coded according to their $g(x)$ value. (Subh83, $a, b)$

for many problems and can significantly speed up the search. As Dijkstra's algorithm, A* is guaranteed to find the optimal path but only if $h(x)$ is admissible and consistent. A heuristic $h(x)$ is said to be

Admissible if for all nodes $x$, the heuristic function $h(x) \leq h^{*}(x)$

Consistent if for all nodes $x$ and all its successor states $x^{\prime}$, the heuristic function $h(x) \leq l(x, u)+h\left(x^{\prime}\right)$

The admissibility criterion means that $h(x)$ never overestimates the true cost of traveling from start to goal and the consistency criterion means that the heuristic cost from a node $x$ can never be larger than the expansion cost $l(x, u)$ plus the heuristics cost to the goal from the expanded node $x^{\prime}$, i.e. the triangle inequality must hold. If a heuristic is consistent it is also admissible but an admissible heuristic is not guaranteed to be consistent. If $h(x)$ is not admissible or consistent it is not guaranteed that the path to the node of the first element in $O$ is the shortest possible, hence, when expanded and put in the closed list there is no way to reopen the node if a shorter path exists and the optimal path can be pruned from the search.

An example of an admissible and consistent heuristic for the point robot in Figure 2.4 is the Euclidean distance. The Euclidean distance will give the shortest possible distance between two nodes so introducing obstacles can only increase the cost. Using this heuristic when no obstacles are present would drive the search straight towards the goal and when comparing to Dijkstra's algorithm which will expand nodes in a circular pattern, 
far fewer nodes are explored. Figure 2.5 illustrates the difference in the node expansion pattern between Dijkstra and $A^{*}$ for a simple scenario with an obstacle and clearly $A^{*}$ expands far less nodes. Since Dijkstra and $\mathrm{A}^{*}$ use a grid to decompose the $\mathcal{C}$-space they only offer resolution completeness and the quality of the path depends on the granularity of the grid. A big problem with grids and search based algorithms is the exponential explosion in the number of nodes when the dimensionality of the problem increases and the time to find a solution quickly becomes intractable.

\subsubsection{Probabilistic methods}

Another family of sampling based methods are the probabilistic methods. Instead of fully discretizing the $\mathcal{C}$-space using a grid decomposition like the discrete search based methods, probabilistic methods draws random samples from $\mathcal{C}$ and tries to connect them using a local steering method. These methods were introduced in the late 1990s and solved many high dimensional problems that had been intractable for previous methods. However, they only offer the weaker probabilistic completeness guarantees. There are two main types of probabilistic algorithms that have been widely used, Probabalistic RoadMaps (PRM) and Rapidly exploring Random Trees (RRT). In a similar way as the combinatorial methods, PRMs build a graph structure in $\mathcal{C}$, but instead of fully characterizing $\mathcal{C}_{\text {free }}$ they generate random samples that are tested for collision and connected to produce a roadmap that once constructed can be used for multiple queries from any start to goal within the road map (Kavraki et al., 1996). This section will focus on the singlequery RRT algorithm and the discussion will start with the same problem as discussed in Section 2.2.1.

\section{RRT}

The concept of RRTs was introduced by LaValle (1998) for solving high dimensional and dynamic problems. To quickly understand the basic algorithm, the point robot that can
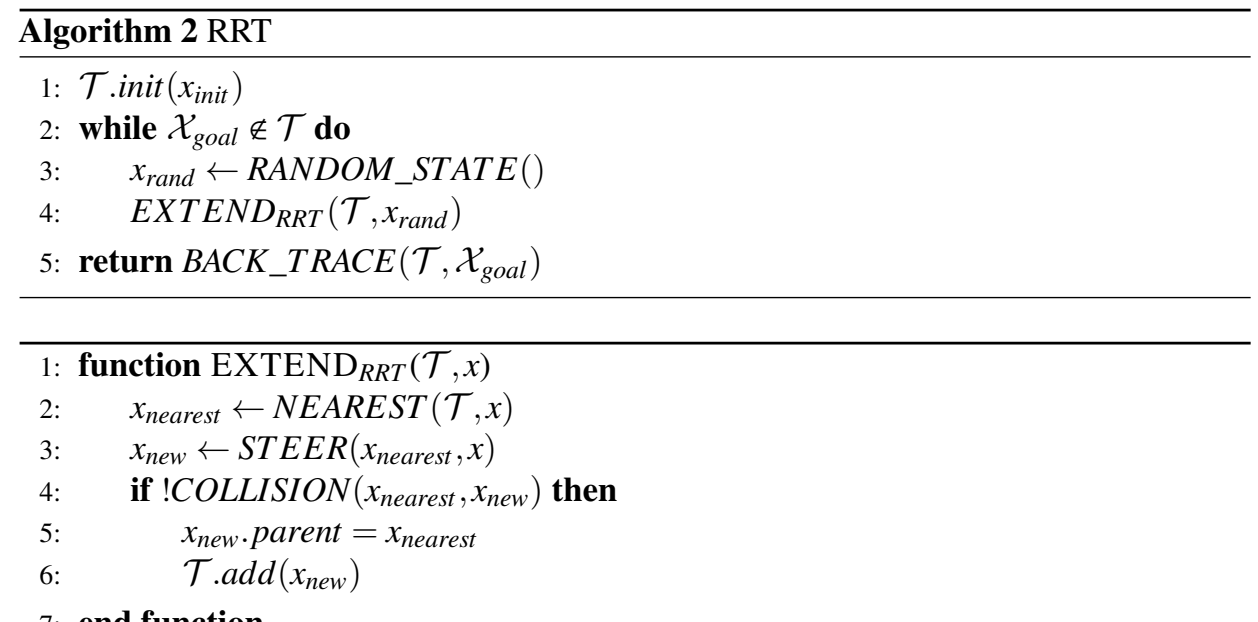

7: end function 


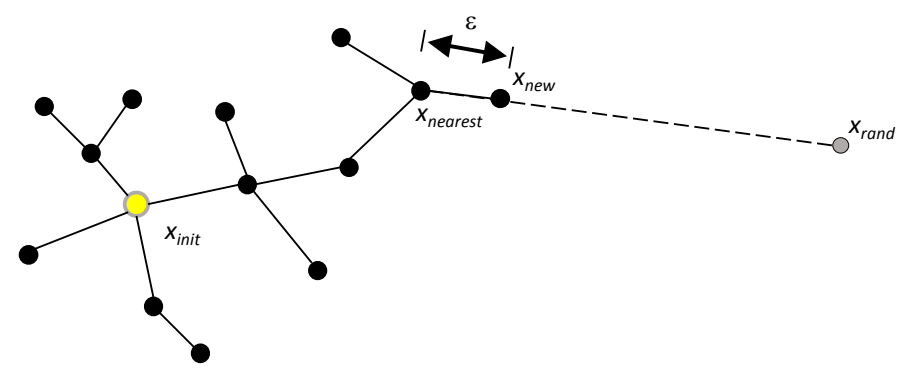

Figure 2.6: Illustration of one step in the RRT iteration. A small step of length $\varepsilon$ is taken along the line between $x_{\text {nearest }}$ and $x_{\text {rand }}$ to generate $x_{\text {new }}$ which is inserted in the tree.

move in any direction will be considered again. Now, instead of being constrained to the grid with only 4 possible movements the robot is allowed to move in any direction. The basic RRT follows a few simple steps and the idea is to get an aggressive exploration of the $\mathcal{C}$-space by probing randomly selected configurations from $\mathcal{C}$. The RRT maintains a tree structure $\mathcal{T}=(\mathcal{V}, \mathcal{E})$ that is a special type of graph where each node only has one parent node. The tree $\mathcal{T}$ is rooted in $x_{\text {init }}$ and in each iteration the tree is expanded by drawing a random configuration from $\mathcal{C}$ and trying to connect it with a collision free edge from the closest node in the tree. Using a uniform distribution over $\mathcal{C}$ to generate the samples results in a bias that pulls new branches of the tree towards unexplored regions. The following procedures are needed to perform one step in the RRT algorithm:

Sampling: Let RANDOM_STATE() be a function that returns independent identically distributed random configurations from $\mathcal{C}$. Here it will be assumed that they are drawn from a uniform distribution but the concept generalizes to other distributions that has dense coverage of $\mathcal{C}$ in the limit.

Nearest neighbor: Given a graph $\mathcal{T}=(\mathcal{V}, \mathcal{E})$ where the vertices $\mathcal{V} \in \mathcal{C}$ and a state $x \in \mathcal{C}$, let $\operatorname{NEAREST}(\mathcal{T}, x)$ be a function that returns the "closest" vertex to $x$ given some distance function, $\rho\left(x, x^{\prime}\right)$. In this section Euclidean distance is assumed for determining closeness but for more complicated spaces it can be hard to define a good metric for distance evaluation.

Steering: Given two points $x, y \in \mathcal{C}$ the $\operatorname{STEER}(x, y)$ function returns a point $z \in \mathcal{C}$ that is "closer" to $y$ than $x$ is. For simplicity line segments between $x$ and $y$ will be assumed for now and the point $z$ will lie on the line segment while $|x-z|<\varepsilon$, meaning it will take a step towards $y$ with length $\varepsilon$.

Collision detection: Given two points $x, y \in \mathcal{C}$ the boolean $\operatorname{COLLISION}(x, y)$ function will return true if the line segment between $x$ and $y$ remains in $\mathcal{C}_{\text {free }}$, and false otherwise. 


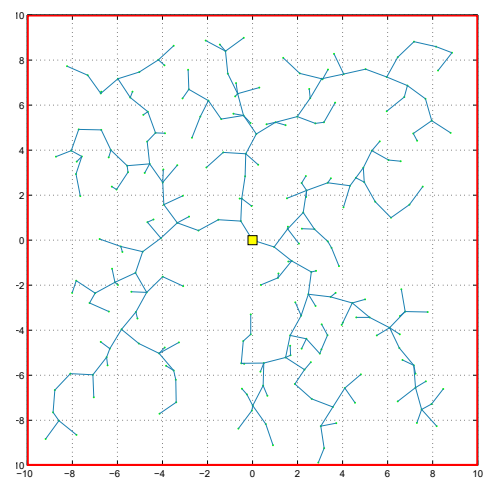

(a)

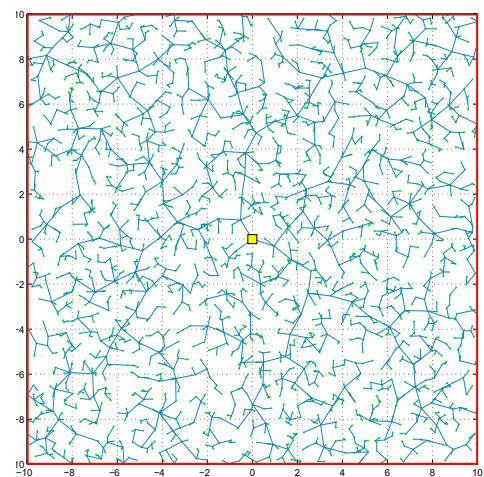

(b)

Figure 2.7: Illustration of the aggressive exploration of an RRT towards unexplored areas. In (a), a tree where 250 vertices have been added is shown and in (b), a tree with 2500 vertices. Image taken from Karaman and Frazzoli (2011) and used with courtesy of the authors.

Given these basic procedures above, the RRT algorithm can now be summarized in Algorithm 2. It enters a loop and continues to expand nodes until a node in the goal region is inserted in the tree. Figure 2.6 illustrates the basic extension step in the RRT and in every iteration a random sample $x_{\text {rand }}$, is drawn from $\mathcal{C}$ using the sampling procedure. In the next step the nearest neighbor, $x_{\text {nearest }}$, to $x_{\text {rand }}$ in the tree is found. Using a spacepartitioning data structure such as a kd-tree for nearest neighbor queries significantly reduces the run-time time and give a logarithmic complexity in the number of nodes (Friedman et al., 1977). Once $x_{\text {nearest }}$ has been established a step of length $\varepsilon$ is taken along the line between $x_{\text {nearest }}$ and $x_{\text {rand }}$ which is checked for collisions before inserted in the tree and the steps are repeated.

Figure 2.7 illustrates the aggressive exploration achieved by the RRT. The first few samples quickly branch out to reach all corners and as more samples are added the space becomes more densely sampled. The probability of connecting a sample in $\mathcal{X}_{\text {goal }}$, if a valid path exists, tends to one as the number of samples approaches infinity and the RRT algorithm is therefore probabilistically complete, however there have not yet been any theoretical result that establishes the rate of convergence. If no solution exists the algorithm will run forever which is one of the drawbacks with probabilistically complete algorithms. Even though they offer only weak guarantees they have proved to solve many practical problems, but in environments with many narrow passages, slow convergence is a problem. Slightly modifying the sampling function so $x_{\text {goal }}$ is selected with a specified probability or building bi-directional trees, one rooted in $x_{\text {init }}$ and one in $x_{\text {goal }}$, can improve performance in some applications (LaValle et al., 2000). 


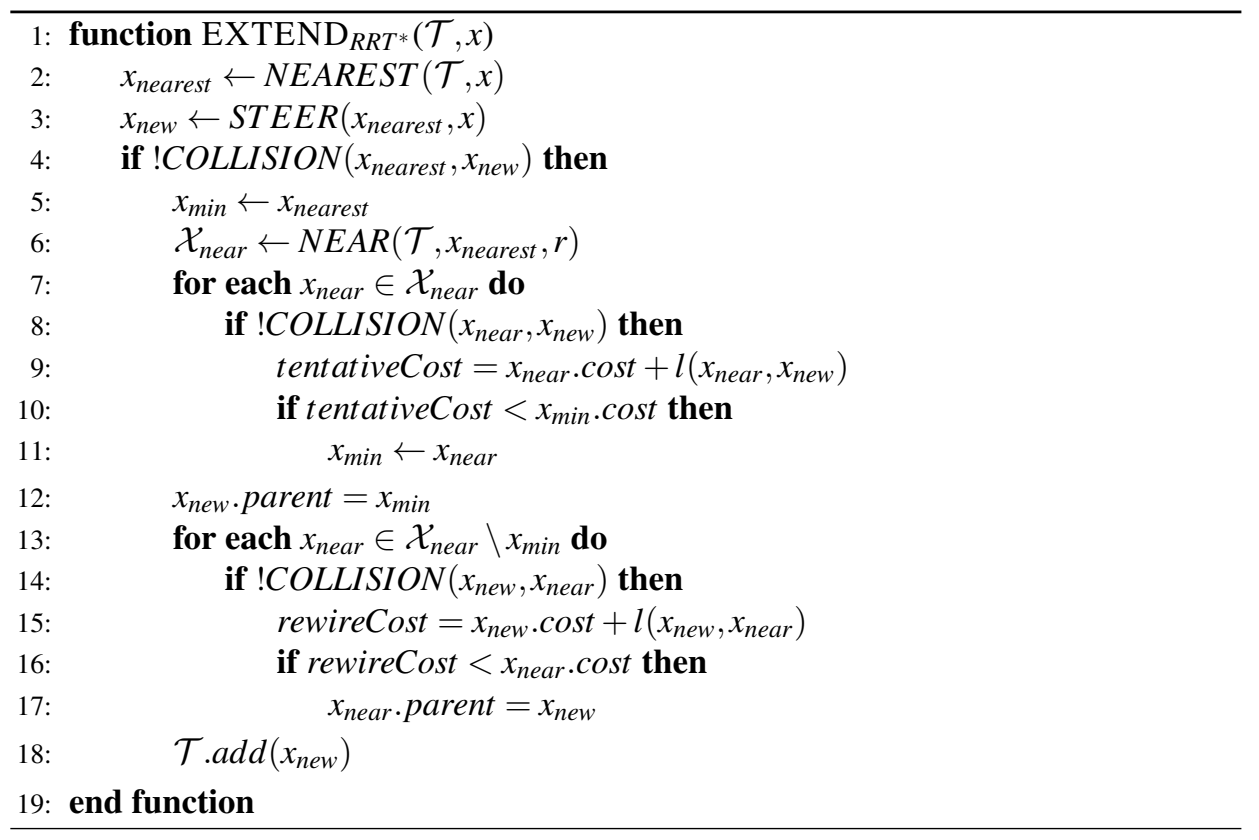

\section{RRT* $^{*}$}

RRTs can find solutions to many problems but there are no guarantees on the quality of the solution. Karaman and Frazzoli (2010a) even show that the RRT algorithm converges almost surely to a sub-optimal solution but in the same work they also present an extension to the RRT framework that gives the algorithm probabilistic optimality meaning it will converge to the optimal solution when the number of samples approaches infinity. They call the extended version RRT* and it only involves minor modifications to the extend function in the original formulation in Algorithm 2. A new procedure is introduced for the extension:

Near nodes: Given a graph $\mathcal{T}=(\mathcal{V}, \mathcal{E})$ where the vertices $\mathcal{V} \in \mathcal{C}$, a state $x \in \mathcal{C}$ and a positive real number $r$, let $\operatorname{NEAR}(\mathcal{T}, x, r)$ be a function that returns all the nodes, $\mathcal{X}_{\text {near }}$, contained in a ball around $x$ with radius $r$.

Given this the extend function is modified as described in EXTEND $R R T^{*}$ and replaces the EXTEND $D_{R R T}$ in Algorithm 2. The function begins with the exact same steps, first it expands a node $x_{\text {new }}$ along the line between $x_{\text {nearest }}$ and $x_{\text {rand }}$ as seen in Figure 2.8a. The next step, illustrated in Figure 2.8b, differs from the original RRT, instead of directly connecting $x_{\text {new }}$ to the nearest node $x_{\text {nearest }}$, the $\operatorname{NEAR}(\mathcal{T}, x, r)$ function is called to find all nodes $\mathcal{X}_{\text {near }}$ that lie in a ball with radius $r$ around $x_{\text {new }}$. The node $x_{\text {new }}$ is then connected to the node $x_{\text {min }} \in \mathcal{X}_{\text {near }}$ that renders the overall lowest cost from $x_{\text {init }}$ to $x_{\text {new }}$. The last step, shown in Figure 2.8c, rewires the tree if any of the nodes in $\mathcal{X}_{\text {near }}$ benefits by rewiring its parent connection through $x_{\text {new }}$. As more nodes are added the radius is reduced as $r=\min \left\{\left(\frac{\gamma}{\zeta_{d}} \frac{\log |\mathcal{V}|}{|\mathcal{V}|}\right)^{1 / d}, \varepsilon\right\}$ where $d$ is the dimension of the configuration space, $\gamma$ is a 


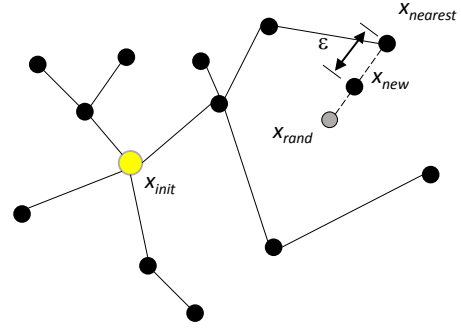

(a)

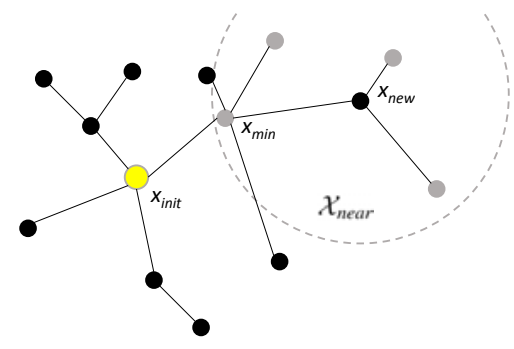

(c)

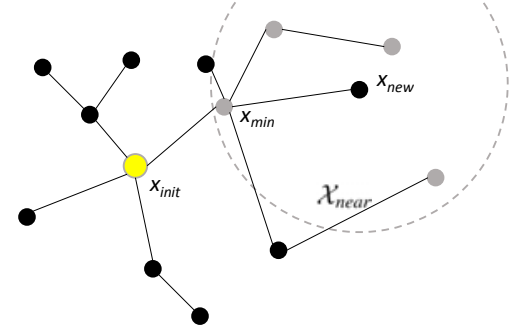

(b)

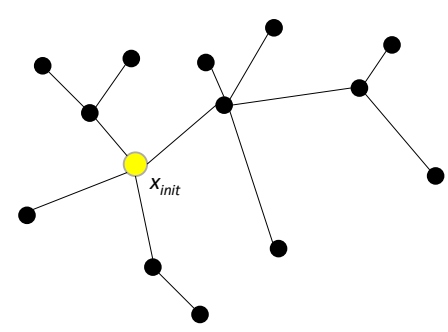

(d)

Figure 2.8: Illustration of the extra steps performed by the $R R T^{*}$ algorithm. In (a) the original step to produce $x_{\text {new }}$ is taken. In (b) the gray nodes represent the nodes in $\mathcal{X}_{\text {near }}$ and $x_{n e w}$ is connected to the node in $\mathcal{X}_{\text {near }}$ that renders the best cost from $x_{\text {init }}$ to $x_{\text {new }}$. In (c) all the nodes that benefit from rewiring its parent through $x_{\text {new }}$ does so to render the new optimized tree in (d).

constant and $\zeta_{d}$ is the volume of the unit ball of dimension $d$. These simple extensions incur little extra computation time compared to the original RRT but they result in significantly better paths than the original formulation. Since the tree is constantly optimized and rewired the optimal solution will be found when the number of samples approaches infinity and the algorithm is said to be probabilistically optimal. Further proofs and arguments of this property can be found in work by Karaman and Frazzoli (2010a). Figure 2.9 shows the expansion of the RRT and RRT* algorithms in a cluttered environment when 1000, 2500 and 15000 iterations have been performed. Both algorithms find a suboptimal solution quickly. However, as the number of samples increase, the RRT solution hardly changes while the RRT* algorithm constantly improves the result and with 15000 samples, a good approximation of the two straight line segments constituting the optimal solution can be seen. Many modifications to the original formulations can be found in the literature and pruning techniques and different heuristic functions that biases the sampling to interesting regions have been used to speed up the search but they are outside the scope of this thesis (Urmson and Simmons, 2003; Nasir et al., 2013; Kalisiak and van de Panne, 2006; Gammell et al.). 


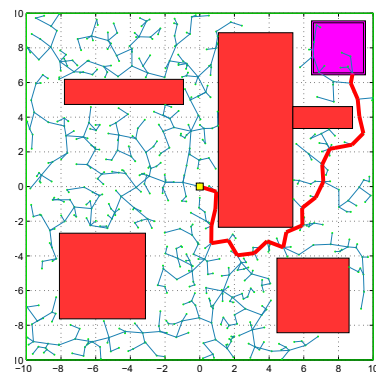

(a)

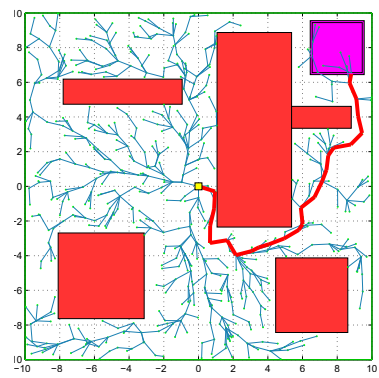

(d)

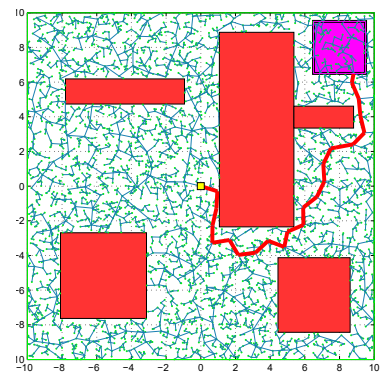

(b)

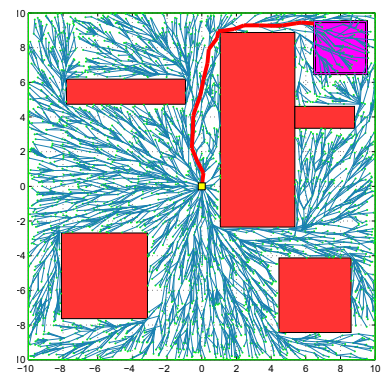

(e)

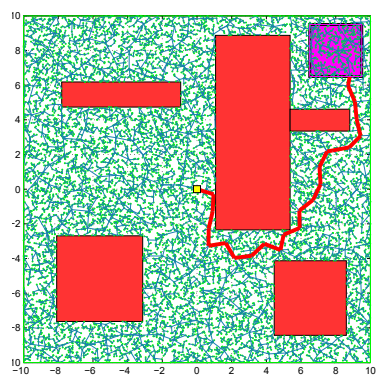

(c)

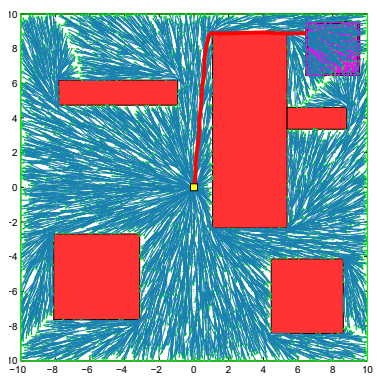

(f)

Figure 2.9: Comparison between the solution found by $R R T$ and $R R T^{*}$ algorithms after, (a) and (d), 1000 iterations, (b) and (e), 5000 iterations and (c) and (f), 15000 iterations. Top row shows results from the original RRT and the bottom row shows results from $R R T^{*}$. The solution found by $R R T^{*}$ is clearly superior to the solution found by the original RRT. Image taken from Karaman and Frazzoli (2011) and is used with courtesy of the authors. 


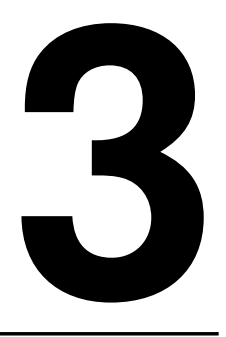

\section{Motion planning for autonomous vehicles}

The previous chapter introduced the classical motion planning problem for holonomic platforms that can move in any direction. The paths and control inputs generated from the classical algorithms are often unsuitable for direct applications to a robot or vehicle that is kinematically constrained. Therefore a special treatment for non-holonomic platforms with differential constraints are needed. This chapter will introduce the problems related to non-holonomic platforms and give some common approaches of how these can be handled within the frameworks presented in the previous chapter. Focus will primarily be on vehicle models used for autonomous driving and the common separation between unstructured and structured areas will be introduced. The case with moving obstacles that require time to be considered and dynamic environments that continuously change as more information is gained from sensors will also be considered.

A typical execution of a robot task is often abstracted into the old sense-plan-act paradigm that iteratively follows a three step procedure that first gains more knowledge about the environment, then constructs a full or partial plan of how to achieve a goal and finally the plan is acted or executed by the robot. Modern architectures for mobile robots are based on the same steps and, as described by LaValle (2006), a typical plan is devised through a series of steps as shown in Figure 3.1. The first step calculates a collision free path from start to goal. The next step smooths the path in order to conform with the differential constraints of the robot. The piecewise linear paths constructed by the planners in the previous chapter cannot be followed by a car-like vehicle due to the sharp corners and the curvature restriction of a car, so it has to be modified. The third step compute a velocity profile over the path to get a time-parametrized trajectory that does not violate lateral acceleration limits or other comfort or safety constraints. Finally, the last step uses feedback controllers to calculate a control input that makes sure the robot does not deviate too much from the given trajectory.

Ideally a planner that performs all the steps in one go is preferred but is often intractable for most problems with limited computation time. However, efficient planning under differential constraints often result in algorithms that perform some of these steps 
Model of the world

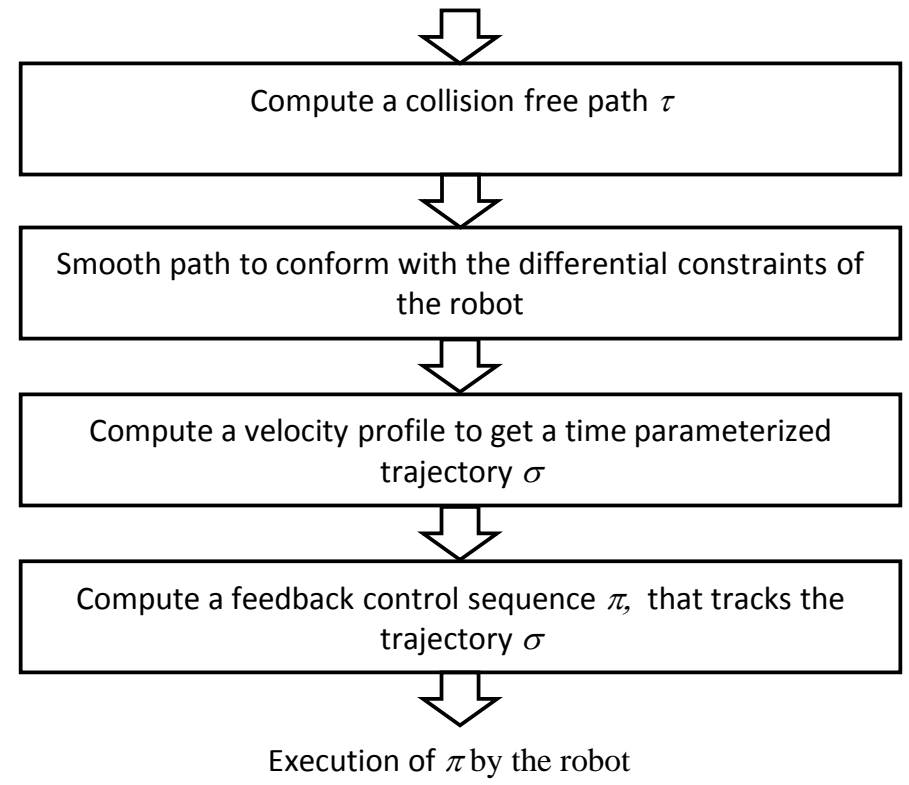

Figure 3.1: The essential steps that need to be performed in each planning cycle before a plan is ready for execution by the robot.

in one go and account for the constraints while constructing the plan. Before presenting planning under differential constraints some tools and models required by the planning frameworks are presented.

\subsection{Differential constraints}

Differential constraints mostly arise from under-actuated systems, i.e., systems that have fewer inputs than the dimension of the configuration space $\mathcal{C}$. They are local constraints that restrict the allowable velocities at every point by forcing them to follow a differential equation. The motion planning problem is lifted from the configuration space $\mathcal{C}$, which only considers static configurations, to the more general state space $\mathcal{X}$ where $\mathcal{C} \subset \mathcal{X}$. Let $q$ represent a configuration of a robot. A first-order differential constraint is then defined as $\dot{q}=f(q, u)$ and a second order constraint as $\ddot{q}=f(q, \dot{q}, u)$ where $u \in \mathcal{U}$ represents the input to the system. First order constraints are often called kinematic constraints and only consider velocity and not the full dynamics of the platform which result in what is called non-holonomic planning. Including the dynamics of a robot results in second order differential constraints and allows for the modeling of acceleration and planning with such models is called kinodynamic planning. Higher-order constraints can be included but here the focus will be on non-holonomic planning for a car-like platform. 


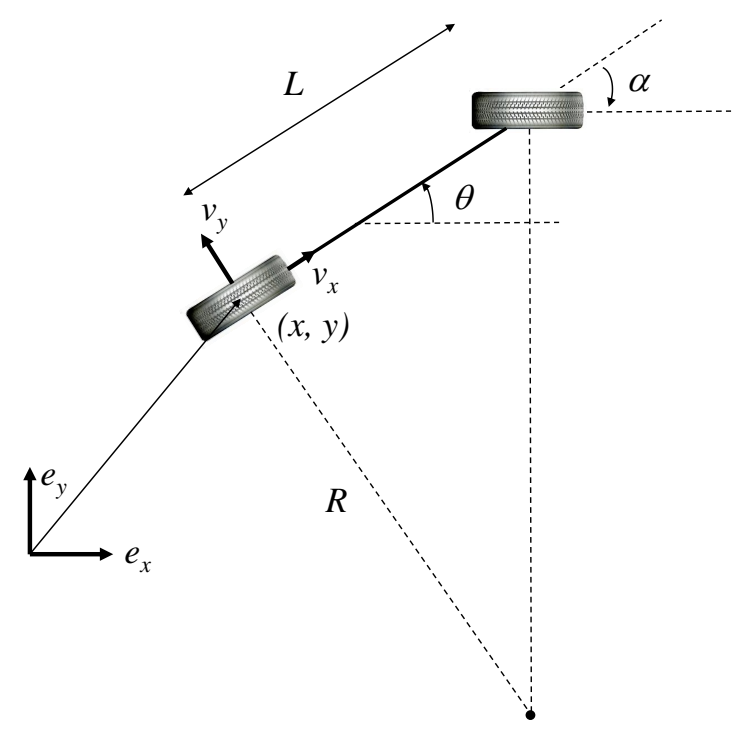

Figure 3.2: Geometry of the basic single track or bicycle model.

A simple yet useful model of a car that does not consider dynamics is the single track model, also known as the bicycle model, shown in Figure 3.2. Consider the case where a car with wheel base $L$ moves forward with velocity $v_{x}$ with a constant steering angle $\alpha$ and assuming no wheel slip, then the car will move along a circle with radius $\mathrm{R}$. The kinematic constraints can then be derived by trigonometry. Let $q=(x, y, \theta)$, where $(x, y)$ represents the position of the rear axle of the car and $\theta$ the heading of the car. From Figure 3.2 it can be seen that the corresponding velocity vector $\dot{q}=(\dot{x}, \dot{y}, \dot{\theta})$ is constrained to $\dot{x} / \dot{y}=\tan (\theta)$, which together with the constraint $R=L / \tan (\alpha)$ gives the following first order differential constraints:

$$
\begin{array}{r}
\dot{x}=v_{x} \cos (\theta) \\
\dot{y}=v_{x} \sin (\theta) \\
\dot{\theta}=\frac{v_{x}}{L} \tan (\alpha)
\end{array}
$$

Setting the maximum steering angle $\alpha<\left|\alpha_{\text {max }}\right|$ results in a minimum turning radius $R_{\text {min }}$ and it is now clear that this model is non-holonomic since it is impossible to move sideways without violating the no slip condition. Restricting the allowed velocities and steering angles to the finite set of numbers $\mathcal{U}_{v_{x}}=\{0,1\}$ and $\mathcal{U}_{\alpha}=\left\{-\alpha_{\max }, 0, \alpha_{\text {max }}\right\}$ results in the Dubin's car that can only stop and move forward at unit speed (Dubins, 1957) and setting it to $\mathcal{U}_{v_{x}}=\{-1,0,1\}$ results in the Reed-Shepp car that can also reverse at unit speed (Reeds and Shepp, 1990). Even though these models are very simplified they have efficient analytic solutions for optimal paths between any two states that can be useful for designing heuristic functions for search-based motion planners. 
A time discretized single-track model is easily obtained using Euler forward or higher order methods. By integrating the differential equations forward in time a simulated path or trajectory resulting from a given input can be obtained and used to construct motion segments within a planning framework.

\subsubsection{Two-point boundary value problem}

Given a model $\dot{x}=f(x, u)$ many motion planning frameworks require a method to connect small motion segments between two nearby states. Some simpler models have efficient analytic solutions that are easily incorporated in motion planning frameworks. However, most interesting models do not have this property and numerical optimization techniques from optimal control is needed to get a solution. The problem of connecting a start state to a final state is an optimal control problem called a Two Point Boundary Value Problem (TPBVP) and is a small planning problem in itself and can often be very complicated even in the obstacle-free case.

Given the model $\dot{x}=f(x, u)$ the problem can be formulated as

$$
\underset{u(t)}{\operatorname{minimize}} \int_{0}^{t_{f}} \phi(x(t), u(t)) d t
$$

subject to

$$
\begin{aligned}
\dot{x} & =f(x, u) \\
h(x, u) & \leq 0 \\
x(0) & =x_{\text {init }} \\
x\left(t_{f}\right) & =x_{\text {goal }}
\end{aligned}
$$

where $\phi(x(t), u(t))$ is a cost function, $h(x, u)$ represents the inequality constraint functions on state variables $x$ and control inputs $u$. Furthermore, $x_{\text {init }}$ and $x_{\text {goal }}$ are the initial and end states, respectively. The formulation is very similar to the classical motion planning problem but here the focus is to find a solution that respects the differential constraints $\dot{x}=f(x, u)$ directly. Depending on the complexity of $f(x, u)$, even the simple formulation without any obstacles can become intractable. By formulating a discrete-time model $x_{k+1}=f\left(x_{k}, u_{k}\right)$ numerical optimization techniques can sometimes be applied to find a local solution the problem. Collocation and shooting methods where the models are simulated forward using a discretized or parameterized control input can be used together with Newton methods to iteratively find a solution to the problem. Figure 3.3 shows a collection of solutions from different start and goal states for a reversing truck and trailer system described in Paper B and C.

If the problem formulation is convex a global optimum can be obtained otherwise the methods might get trapped in a local optimum. In this thesis we see these methods as a tool and efficient software packages such as ACADO (Houska et al., 2011) are available. For a deeper understanding in numerical optimization techniques the reader is refereed to (Nocedal and Wright J., 2006) and for optimal control the reader is refereed to (Betts, 2010). 


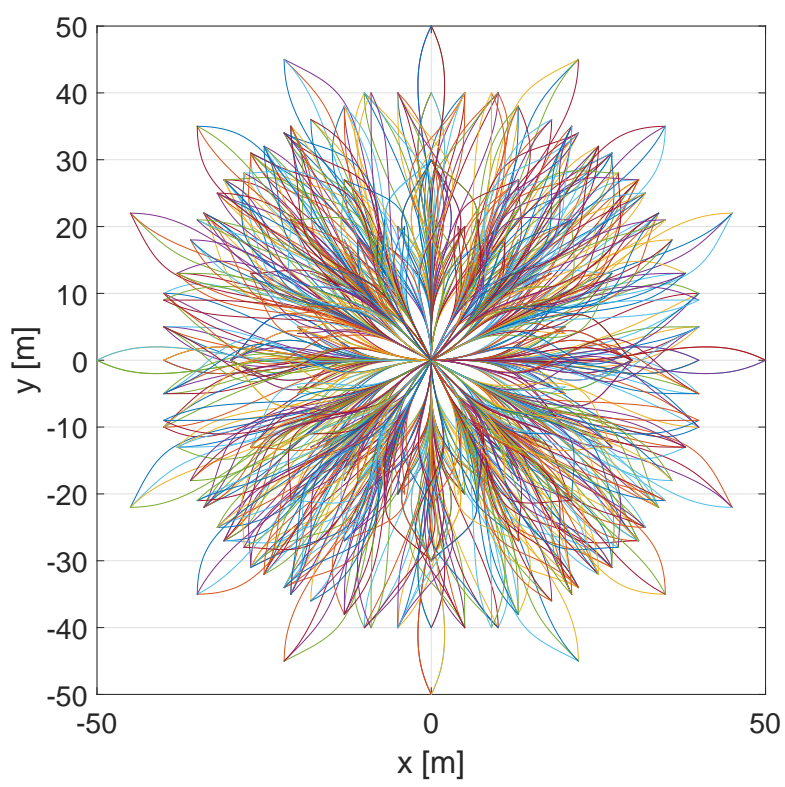

Figure 3.3: A collection of motion segments for a general 2-trailer. Solutions are calculated using ACADO from the origin with several different starting headings to different end positions and headings. Symmetry is exploited so calculations only need to be performed in the first quadrant and then the solutions are mirrored into the other quadrants.

\subsection{Planning under differential constraints}

Planning under differential constraints is conceptually no different from the classical formulation. The problem is formulated in the same way but in the state space $\mathcal{X}$ instead of $\mathcal{C}$. Find a control input $u \in \mathcal{U}$ that transfers the system $\dot{x}=f(x, u)$ from an initial state $x_{\text {init }}$ to $x_{\text {goal }}$ while $x \in \mathcal{X}_{\text {free }}$. A challenging aspect when considering second-order differential constraints is the drift introduced when modeling acceleration constraints. Even if a vehicle is in $\mathcal{X}_{\text {free }}$ there might be no available control input $u \in \mathcal{U}$ that will keep $x \in \mathcal{X}_{\text {free }}$ when time progresses due to the momentum of the car. This region is called the region of inevitable collision $\mathcal{X}_{\text {ric }}$ and is problematic because it can be very hard to characterize. There is no simple test, such as the collision detection method, that can determine if a state is in $\mathcal{X}_{\text {ric }}$ or not. Therefore, planning algorithms usually only consider $\mathcal{X}_{o b s}$ which can cause the algorithm to get trapped and waste a lot of exploration in parts of the state space that is in $\mathcal{X}_{\text {ric }}$.

When considering autonomous vehicles, the planning problem is usually divided into two different situations that often need different approaches. The first is the unstructured area such as parking lots or other open areas where rules are less specified and speeds are 


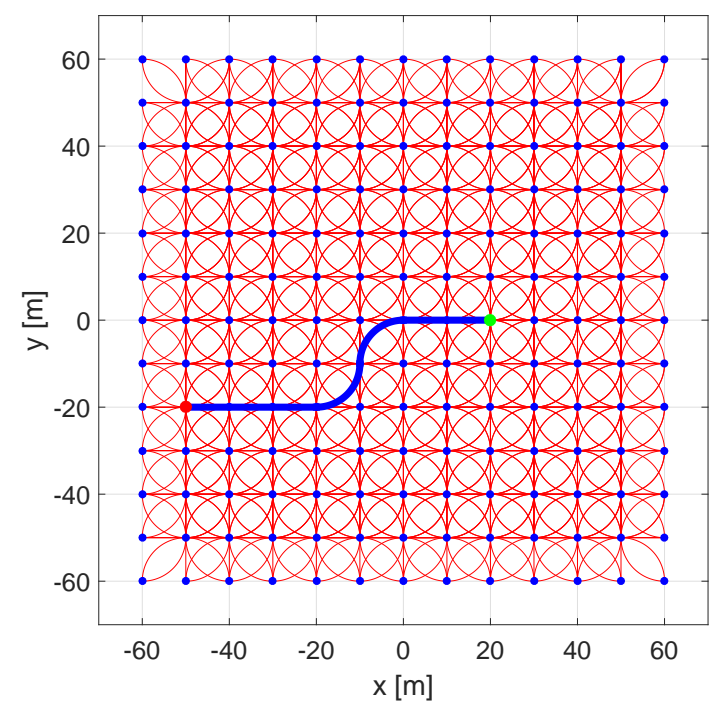

(a)

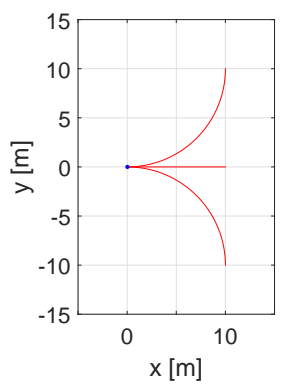

(b)

Figure 3.4: A state lattice produced for the Dubin's car with the possible motions shown in (b). Repeatedly applying these motions give the state lattice shown in (a). A shortest path that conforms with the motion model for Dubin's car can now be found through a search on the grid. The blue path shows the shortest path between the red and green points in the grid.

lower. Here the classical sampling-based techniques can be modified to conform with the kinematic constraints of the vehicle and efficient methods have been developed. The other situation is structured areas where well-defined rules are present and speeds can be higher. The road network has well-defined rules and a known structure which makes it possible to make assumptions that can drastically reduce the search space. However, moving vehicles, pedestrians, erratic drivers and the social interplay between human drivers significantly complicates the problem and at the moment a lot of research is focused on how to model and handle this uncertainty for urban driving scenarios.

\subsubsection{Unstructured areas}

Almost all practical algorithms for planning in unstructured areas under differential constraints are sampling-based and the same principles as presented in Chapter 2 can be used to solve the problem. A common approach for the discrete search-based methods is to discretize parts of the state space in the same way that the configuration space was discretized for the classical methods. However, since the motion model $\dot{x}=f(x, u)$ is a continuous function, the input $u$ also needs to be discretizised in order to apply search based methods. Let $u_{d} \in \mathcal{U}_{d} \subset \mathcal{U}$ where $\mathcal{U}_{d}$ is a finite subset of the possible inputs in $\mathcal{U}$. The model $\dot{x}=f(x, u)$ can then be used as an incremental simulator to generate a trajectory 
that together with a collision detection function can be used to determine if a trajectory segment is valid or not. The state space can then be explored and probed by applying the available inputs in $\mathcal{U}_{d}$ to build a tree of possible input sequences. Using a TPBVP solver to find the inputs $\mathcal{U}_{d}$ that solve the problem from one grid point to another it is possible to create motion primitives that trap the tree on a lattice structure that is easily searched by $\mathrm{A}^{*}$ or other search methods. Figure 3.4 shows an example for the Dubin's car model where a coarse discretisation of $10 \mathrm{~m}$ and four different possible headings following the cardinal directions are used. Every search step has three possible actions, go straight forward, turn maximum right and turn maximum left and by iteratively applying these actions the state lattice is formed. For more complex motion models the time required to solve the TPBVP problem in every extension quickly makes this strategy infeasible, so instead the possible actions are calculated offline to create a library of motion primitives, where each motion primitive represents a given input and can be accessed quickly during the search. Parts of such a library for a reversing general 2-trailer is shown in Figure 3.3 and compared to the simple example with the Dubin's car that only had three possible actions these motion primitives allow for a much smoother set of movements but at the cost of extra search time.

When executing a trajectory, it is important that the whole trajectory is physically possible to execute. Even if the motion primitives are derived through the model $f(x, u)$ it is not guaranteed that there is a smooth connection at the grid points or that an instant switch between two inputs in $\mathcal{U}_{d}$ is possible. A desired property is to have a continuous curvature along the trajectory and this can either be achieved when solving the TPBVP by forcing the curvature to values that are possible to connect at the intersection points or by running a post-optimization step that smooths the trajectory found by the search algorithm. For the example with the Dubin's car an instant movement of the steering wheel is needed at the intersection points which is clearly not physically possible and plans generated with the Dubin's car are not inherently executable. Lattice-based planners offer resolution completeness that depends on the granularity of the lattice, so a trade-off between solution quality and planning speed has to be done. The lattice approach has proven to be very effective and demonstrated on several implementations for autonomous robots including the DARPA Urban challenge winner (Urmson et al., 2008), on Mars rovers (Pivtoraiko et al., 2009) and other industrial applications (Cirillo et al., 2014).

\section{Heuristics}

With the increasing number of possible actions in the motion primitive library, a good heuristic function is essential to reach acceptable performance. However, the difficulty of constructing one increases when differential constraints are introduced. Euclidean distance was efficient and easy to calculate for the problems in Chapter 2 but can be very misinformative in many cases when non-holonomic platforms are considered. For instance, consider the case when calculating an estimate of moving a car from a given position to a new location a short distance straight to the left or right. Using the Euclidean distance as the heuristic, nodes that are geometrically close would look promising for expansion even though it would be impossible, due to the differential constraints, to move the car to the new location without first covering the much longer distance by moving forward and then reversing back into the new location. Figure 3.5 shows the difference in covered 


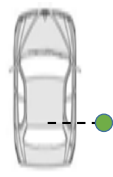

(a)

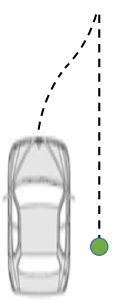

(b)

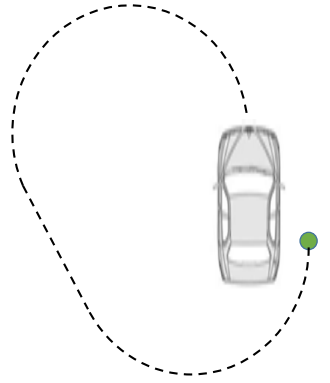

(c)

Figure 3.5: Comparison of the estimated heuristic distance for moving a car a short distance straight to the right. (a): Euclidean distance. (b): Distance covered by Reed-Shepp car. (c): Distance covered by Dubin's car.

distance between the straight line, Dubin's car and Reed-Shepp car when calculating the distance for this maneuver. Choosing bad heuristics causes the planner to wastefully expand nodes that look promising according to the heuristic but are in reality bad candidates for exploration. A common approach when designing heuristic functions is to remove parts of the constraints and solve the simpler problem. This is hopefully much faster to solve and gives an underestimate of the true cost. For a car in an unstructured environment an efficient strategy developed by Dolgov et al. (2010), is to first solve the non-holonomic problem but without obstacles, then solve the holonomic problem with obstacles and finally use the max operator between the two to get the heuristic value. As seen in Figure 3.5 a good approximation for the non-holonomic problem without obstacles could be to use the solution to the Reed-Shepp problem since it has a fast analytic solution or precompute a map with shortest distances to all neighboring states in a vicinity around the goal. This heuristic guides the search and expands nodes that are approaching the goal with a suitable heading. It gives better performance than the Euclidean distance alone but since it does not take obstacles into account it can be fooled and explore dead end areas. The second heuristic is designed to avoid dead end areas by first solving the cost-to-go from all points for the $2 \mathrm{D}$ problem by running a wave-front propagation or Dijkstras algorithm until all nodes have been reached. The effect of this heuristic is that it detects all dead ends in the obstacle map and can guide the search of the more expensive nonholonomic planner away from those areas. A problem with heuristically guided search methods is that there exists no general way to generate a good heuristic function and a lot of domain-specific knowledge is needed to construct one, however when a good heuristic function is found they can have excellent practical performance.

\section{Probabilistic methods}

The probabilistic RRT methods discussed in the previous chapter are easily modified to incorporate the differential constraints. As discussed earlier the TPBVP can be hard to solve and even if it is possible to perform offline calculations it might be undesirable to 


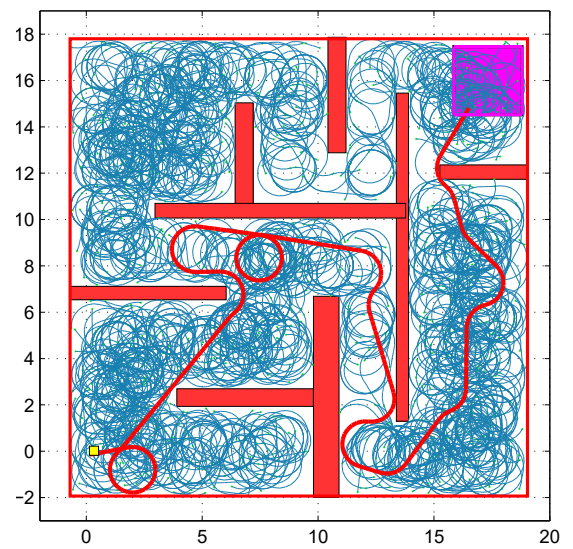

(a)

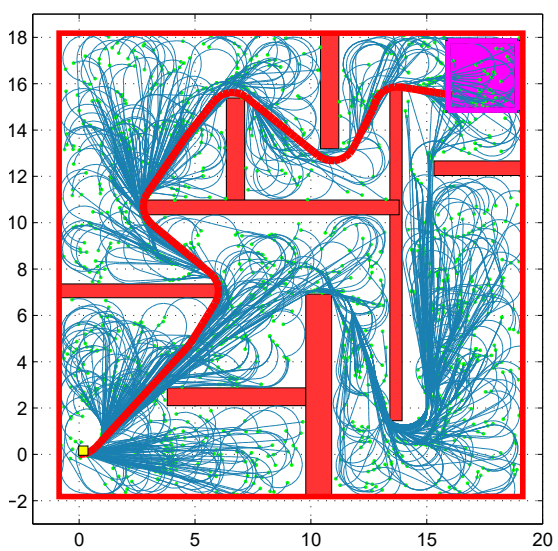

(b)

Figure 3.6: Solutions to a planning problem with Dubin's car for $R R T$ and $R R T^{*}$, respectively. The solution returned from the $R R T$, shown in (a), contains a lot of unnecessary turns and is far from optimal. Using $R R T^{*}$ the much better solution shown in (b) is found. Image taken from Karaman and Frazzoli (2010b) and is used with courtesy of the authors.

store a big library of motion primitives. To avoid the explicit computation of TPBVPs, LaValle and Kuffner (2001) introduced the idea of kinodynamic planning within the RRT framework where the model $\dot{x}=f(x, u)$ is used as an incremental simulator and inputs are randomly chosen so no TPBVP has to be solved. The algorithm follows the same steps as Algorithm 2 with only changes in the distance function $\rho\left(x, x^{\prime}\right)$ and the $\operatorname{STEER}(x, y)$ function. Designing a good distance function with differential constraints experiences the same problems as finding a good heuristic function for the search methods, so the same types of ideas can be used again. Offline computation, Dubin's car or Reed-Shepp car can be used for car-like platforms but the efficiency of the nearest-neighbor search can be compromised since there might be no efficient space-partitioning algorithm for the chosen distance function $\rho\left(x, x^{\prime}\right)$, so a trade-off between distance estimation and implementation efficiency of the nearest neighbor search has to be considered. Once $x_{\text {nearest }}$ is determined, the $\operatorname{STEER}(x, y)$ function is applied. If an exact connection is possible such as with a Dubin's car model, $x_{\text {nearest }}$ is connected to $x_{\text {rand }}$ exactly and inserted in the tree before the process is repeated. Instead, if an exact connection is not possible, either a random input is applied and simulated with the motion model $\dot{x}=f(x, u)$ for $\Delta t$ seconds or all available inputs in $\mathcal{U}_{d}$ are simulated and the one with smallest distance value $\rho\left(x_{\text {new }}, x_{\text {rand }}\right)$ is inserted in the tree. By repeating these steps, the state space is incrementally explored by probing the search space with incremental movements satisfying the differential constraints at the same time as the efficient exploration bias towards unexplored regions of the RRT algorithm is maintained. However, as seen in Figure 3.6a, where an RRT built using Dubin's car is shown, the quality of the solution can be very poor and as shown by Kunz and Stilman (2015) even probabilistic completeness cannot be guaranteed depend- 


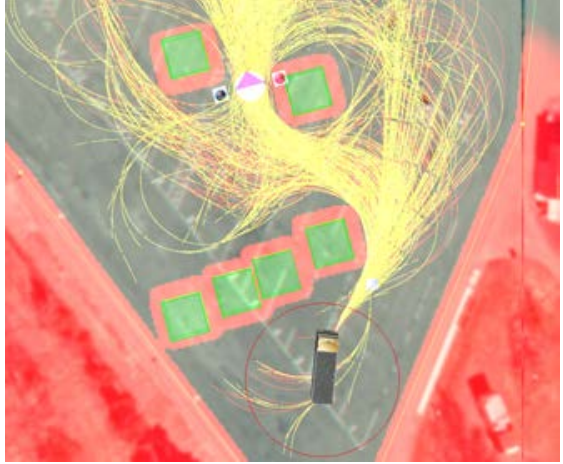

(a)

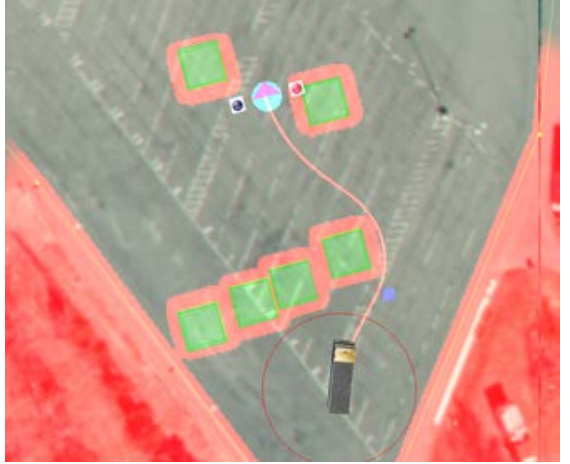

(b)

Figure 3.7: Resulting path from CL-RRT when entering a parking lot. Red areas are not traversable and feasible trajectories stored in the tree are shown in yellow as seen in (a). The shortest path within the tree is shown in (b).

ing on the choice of input extension in the steering function. To increase the quality of the solutions, RRT* can be used to get probabilistically optimal trajectories. However, since several TPBVPs have to be solved in the rewiring step the practical applicability of RRT* $^{*}$ is often limited. For the Dubin's car problem where the exact connections can be done efficiently, RRT* can be applied and the resulting trajectory shown in Figure 3.6b is superior to the one returned by the RRT and is close to the optimal solution.

\section{Deployment}

When deploying these algorithms on real systems many practical concerns emerge that have not been considered in the previous discussions. Perfect environment information has been assumed and no real-time constraints have been considered. Most robot platforms do not have perfect information about their surroundings and instead use sensors to construct a map that is constantly updated as more sensor information is received. This introduces a new problem for planning algorithms as a previously calculated plan can instantly become infeasible due to newly received sensor information and only limited computation time is given to develop a new plan. Many versions of algorithms have been developed that cope with some of these problems. The most straight forward way to handle a dynamically changing map is to simply re-run the planning algorithm at every map update. However, since only small changes of the map are expected, big parts of the search tree can be reused in the replanning step to avoid redundant exploration. The family of $D^{*}$ algorithms originally developed by Stentz (1994) and later refined to the simpler D*-lite by Koenig and Likhachev (2002), keeps the search tree between every iteration and only repairs the affected parts of the tree once the map has changed. When having limited computation time and replanning is expected to be performed several times per second it can also be of interest to first receive a plan of low quality quickly and if available, use the remaining time to improve the plan. Heuristic inflation is often used 
by anytime algorithms, such as ARA* (Anytime A*), to quickly find a low quality plan that can later be improved (Likhachev et al., 2003). Likhachev et al. (2005) developed the Anytime Dynamic $A^{*}$ algorithm that inherits the repairing properties of $\mathrm{D}^{*}$ and the anytime properties of $\mathrm{ARA}^{*}$ by combining the two to get the desired properties of both. Field D* was eventually developed by Ferguson and Stentz (2007) based on these ideas and used successfully to win the DARPA Urban Challenge.

Anytime implementations of RRTs have also been successfully deployed in dynamic environments by Kuwata et al. (2009) and Evestedt et al. (2015) for kinodynamic planning using biased sampling and closed loop steering models. Figure 3.7 shows an instance of an RRT used on a truck when entering a parking lot and the resulting plan to reach the goal.

\subsubsection{Structured areas}

A slightly different problem is faced in structured areas and planning algorithms often need to take a different approach. Instead of finding a path through a geometrically complicated environment, a reference path from the underlying road network is usually given and the problem instead involves computation of trajectories that slightly deviate around the reference while abiding to the driving rules, feel natural and comfortable for a passenger, are safe to execute and give a natural behavior acceptable by other traffic participants. Situations from slow moving dense city traffic with pedestrians and bicycles all the way up to situations at highway speed need to be handled in order to reach full autonomous operation. Controlling speed becomes extremely important since many time-critical traffic situations such as merging and intersection negotiation are frequently encountered. Even though a mining facility presents a less complicated environment than inner city driving, shared public roads are sometimes used, so interaction with other drivers can still be necessary.

A hierarchical planning framework is often used to break down the problem into different abstraction layers in order to make the problem tractable. The top layer or mission planner uses detailed maps of the underlying road network to construct a high-level plan of how to get from A to B. The plan describes which lane to keep and which intersection to turn and delivers a reference path at the center of the intended lane. Detailed mapping of major cities is pursued by many companies and an example of such a map, complete with $3 \mathrm{D}$ information and all lane information is seen in Figure 1.5. Assuming the desired reference path is given from the mission planner numerous methods have been presented that tackle the problem in different ways. Since there is no way to know what will be encountered along the complete route, a local problem is solved on a receding time horizon suitable for the given sensor range and computation time. A typical traffic situation can involve several moving objects and the time evolution of the complete scene needs to be considered at planning time in order to calculate a safe trajectory for the ego vehicle. An example of a time-critical trajectory-planning problem can be visualized with a spacetime graph as in Figure 3.8a, where the gray car in the middle is planning an overtaking maneuver to pass the slower moving car while a faster moving car is approaching in the left lane from behind. The predicted future positions of the two vehicles are represented by the blue and green tunnels that projects the future position of the cars onto an imaginary time axis pointing up from the road surface. The ego vehicle has two options for 


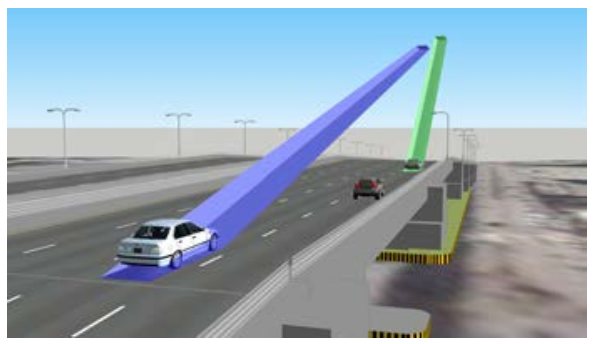

(a)

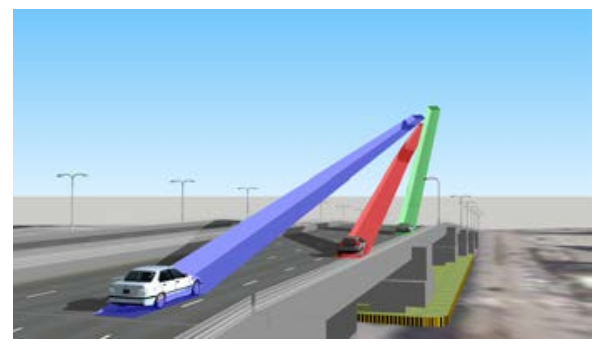

(b)

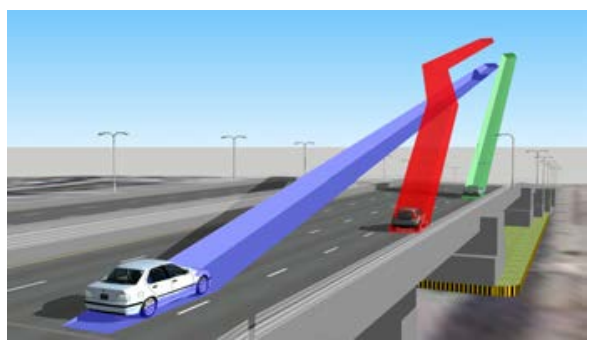

(c)

Figure 3.8: In (a) the gray car in the middle is approaching the green car and wants to perform a lane change to overtake the green vehicle. Future predictions of the movements of the other vehicles are represented by the green and blue tunnels. The two overtaking options for the ego vehicle are illustrated in (b) and (c) by the red tunnels and correspond to the options of either accelerate and overtake before the faster moving vehicle has arrived, (b), or slow down and let it pass before the overtake, (c).

the overtake, either it can accelerate aggressively and overtake the green vehicle before the faster moving vehicle has passed, or it can decelerate and wait for the faster vehicle to pass before executing the overtake. The two different choices are illustrated by the red tunnel in Figure 3.8b and 3.8c, respectively. It is now the planner's task to calculate trajectories that follow the dynamic constraints of the vehicle and determine which action that is the safest and most comfortable option for the passengers in the ego vehicle. There is no general decision on the best way to tackle the problem and it is still an active research field so many customized solutions using different methods have been presented in the literature. Madås et al. (2013) compares three different methods including a lattice based approach and an optimization-based MPC approach but they only consider constant velocity throughout the maneuvers which severely limits the applicability. Kuwata et al. (2009) and Schwesinger et al. (2013) demonstrated the use of probabilistic sampling-based techniques both for a real vehicle and in simulation, but heavily biased sampling is needed to meet real-time constraints and it is difficult to give any guarantees. During the Bertha drive, Ziegler et al. (2014b) used Sequential Quadratic Programming (SQP) to produce smooth trajectories around the center lane but faced problems handling the combinatorial aspects of driving, such as the overtaking decision faced in Figure 3.8. Optimizationbased techniques often require the creation of several convex corridors for each scenario, 
which is a hard task on its own and then several optimization problems for each scenario need to be solved, which can limit the real-time capabilities in complex traffic situations. As seen above, many different techniques, including the ones presented for unstructured planning have been tested for planning in structured driving scenarios and explaining all of them in detail is beyond the scope of this thesis. However, we will give more attention to a method developed by Werling et al. (2011) from which ideas for Paper D have been developed.

\section{Sampling-based trajectory generation}

Given a reference path the problem can be formulated in the Frenet frame where the position of the vehicle is represented by the covered arclength $s(t)$ of the vehicle's perpendicular projection point $\mathbf{r}(s)$ along the reference line and the offset $d(t)$ along the normal at $\mathbf{r}(s)$ as seen in Figure 3.9. The position of the car in the global frame is then given by

$$
\mathbf{x}(s(t), d(t))=\mathbf{r}(s(t))+d(t) \mathbf{n}_{c}(s(t))
$$

where $\mathbf{n}_{c}$ is the normal vector at $\mathbf{r}(s)$. Assuming a reasonably constructed reference path, the lateral and longitudinal motions $d(t)$ and $s(t)$ respectively, can be modeled as two separate integrator systems with $d(t)=x_{1}$ and $s(t)=x_{1}$ given by the system

$$
\dot{x}=\left[\begin{array}{lll}
0 & 1 & 0 \\
0 & 0 & 1 \\
0 & 0 & 0
\end{array}\right] x+\left[\begin{array}{l}
0 \\
0 \\
1
\end{array}\right] u
$$

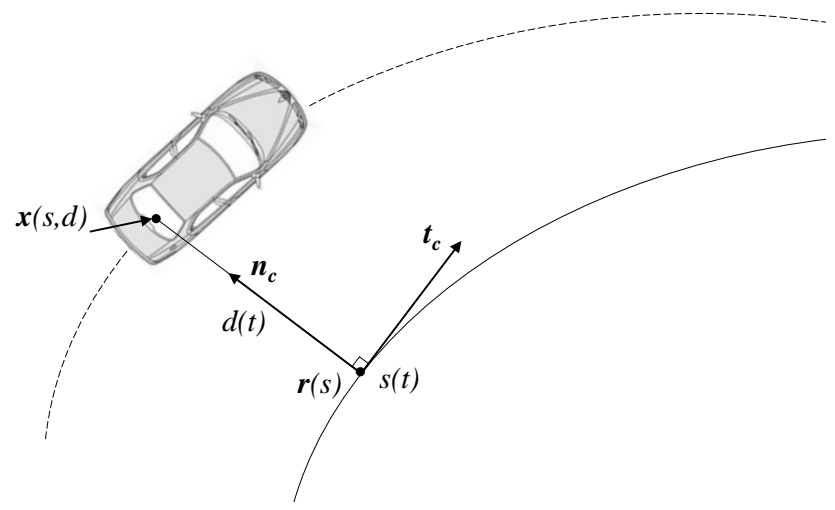

Figure 3.9: Car position represented in the Frenet frame. The solid line represents the reference line and the position of the vehicle is represented by the covered arclength $s(t)$ of the vehicle's perpendicular projection point $\mathbf{r}(s)$ along the reference line and the offset $d(t)$ along the normal at $\mathbf{r}(s)$. 
where $x=\left(x_{1}, x_{2}, x_{3}\right)^{T}$. The input $u$ represents the lateral or longitudinal jerk, $\ddot{d}(t)$ or $\dddot{s}(t)$, respectively. Defining the cost functional

$$
\mathscr{J}=\frac{1}{2} \int_{0}^{t_{f}} u^{2} d t
$$

and solving for the optimal minimum jerk trajectory from an initial state $x_{0}=\left[x_{10}, x_{20}, x_{30}\right]^{T}$ to a final state $x_{f}=\left[x_{1 f}, x_{2 f}, x_{3 f}\right]^{T}$ gives an analytical polynomial solution on the form

$$
x(t)=\left[\begin{array}{cccccc}
1 & t & t^{2} & t^{3} & t^{4} & t^{5} \\
0 & 1 & 2 t & 3 t^{2} & 4 t^{3} & 5 t^{4} \\
0 & 0 & 2 & 6 t & 12 t^{2} & 20 t^{3}
\end{array}\right]\left[\begin{array}{l}
c 1 \\
c 2 \\
c 3 \\
c 4 \\
c 5 \\
c 6
\end{array}\right]
$$

where the coefficients $c_{1-6}$ are determined from the initial condition $x_{0}$, the final condition $x_{f}$ and the final time $t_{f}$. The original optimization problem including collision checking and physical constraints of the vehicle is much more complicated, but using the fast computable analytic solution and creating a reduced set of interesting terminal states, a vast amount of candidate trajectories can be generated that are checked for validity and the best trajectory within the generated set can be chosen for execution. The process is then repeated and if no new constraints appear between iterations, Bellman's principle of optimality ensures that the rest of the previously found solution is found again resulting in an optimal transfer back to the reference. Since an infinite number of trajectories are impossible to handle, a carefully selected terminal set is needed. Given a time horizon $T_{h}$ on which the problem is solved the lateral movement is discretized into a set of terminal end times $\tau_{f}$ and terminal offsets $\delta_{f}$ to which solutions are calculated to give a set $\mathcal{T}_{\text {lat }}$ of possible lateral movements. The terminal states $x_{2}$ and $x_{3}$ are set to zero to generate trajectories that align with the reference at the end of the trajectory. Figure 3.10a shows the result of this process for a set $\tau_{f}$ with five different end times and a set $\delta_{f}$ with five different offsets on both sides of the reference. A set $\mathcal{T}_{\text {long }}$ of longitudinal movements is also created by solving the problem to a final state $x_{f}=\left[x_{2}, x_{3}\right]$ with arbitrary longitudinal end position $s\left(t_{f}\right)$. The same time discretization $\tau_{f}$ is used and a set $v_{f}$ of terminal velocities are selected with the final acceleration $x_{3}$ set to zero as shown in Figure 3.10b. By combining $\mathcal{T}_{\text {lat }}$ and $\mathcal{T}_{\text {long }}$ in all possible ways and transforming back to global coordinates a vast set of trajectories is obtained. This set can then be checked for trajectories violating curvature, lateral and longitudinal acceleration constraints and other physical limitations of the vehicle. The trajectories are also checked against all static obstacles and against the predicted positions of other traffic participants in both space and time. Once all valid trajectories are determined they are scored according to a cost function that can incorporate comfort, safety and progress i.e.

$$
J[x(t)]=j_{\text {progress }}+j_{a_{\text {lat }}}+j_{\text {acc }}+j_{\text {proximity }}
$$

where the cost term $j_{\text {progress }}$ penalizes trajectories that make slow progress along the reference while $j_{a_{l a t}}$ and $j_{a c c}$ work against the progress terms and reward comfortable smooth 


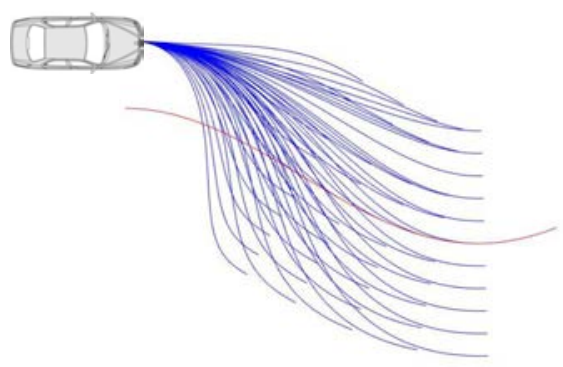

(a)
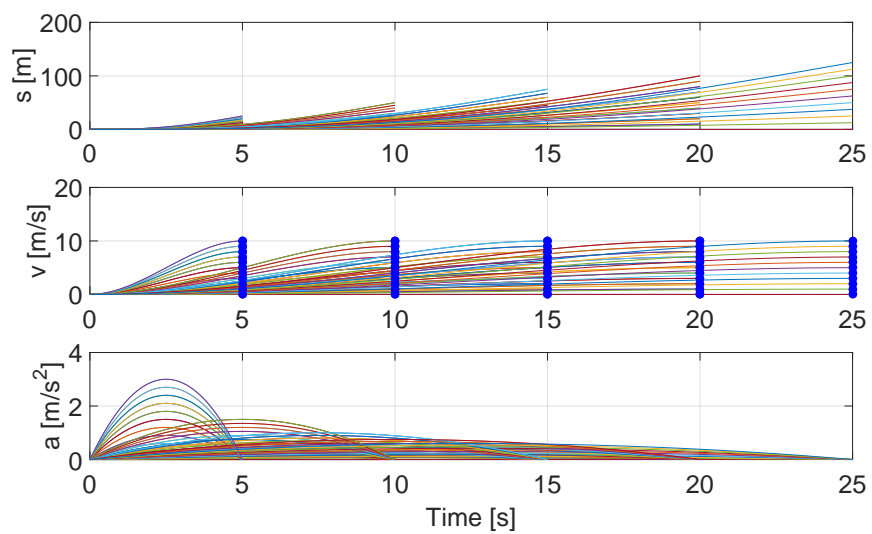

(b)

Figure 3.10: (a): Several candidate paths with different end times $t_{f}$ and different end offsets $d\left(t_{f}\right)$ are calculated and wrapped around the red reference line. (b): Several candidate velocity profiles with different end times $t_{f}$ and different end velocities $v\left(t_{f}\right)$ are generated. Combining them in all possible ways produces a set of trajectories that can be searched to determine the most suitable one.

trajectories with small lateral and longitudinal accelerations. The last term $j_{\text {proximity }}$ puts extra cost on trajectories with close proximity to obstacles to leave a margin for error.

Figure 3.11 shows a situation where a truck is encountering a set of static obstacles illustrated as white boxes. The generated trajectories that did not end up in collision are projected on the road surface and colored according to the evaluated cost and the thick green trajectory represents the selected one with the overall lowest cost. The truck approaches the first obstacle at a speed of $10 \mathrm{~m} / \mathrm{s}$ and gently decreases the speed due to the proximity of the obstacle. It then maintains a low speed throughout the obstacle region before starting to accelerate when the obstacles have been cleared. Already with a naive implementation of the algorithm it is possible to evaluate several thousand trajectories in the order of hundreds of milliseconds, giving real-time capabilities. When used in practice 


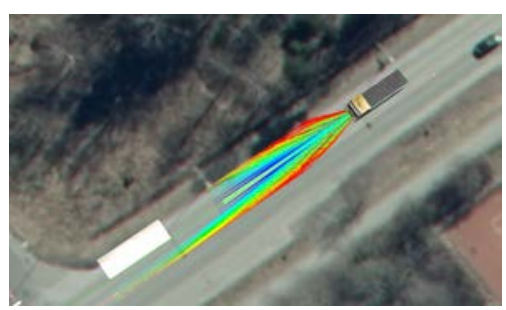

$V=10 \mathrm{~m} / \mathrm{s}$

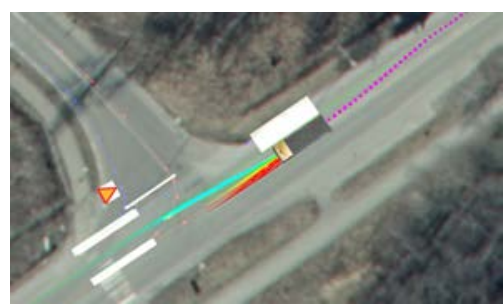

$v=4.4 \mathrm{~m} / \mathrm{s}$

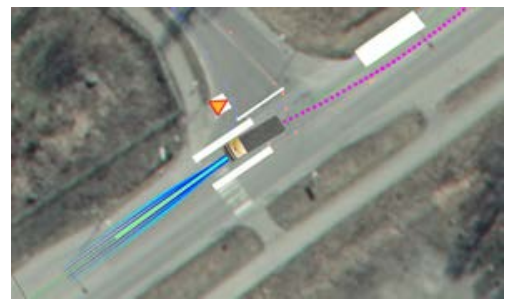

$v=2.6 \mathrm{~m} / \mathrm{s}$

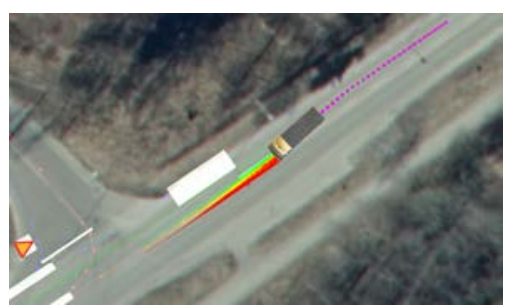

$v=3.4 \mathrm{~m} / \mathrm{s}$

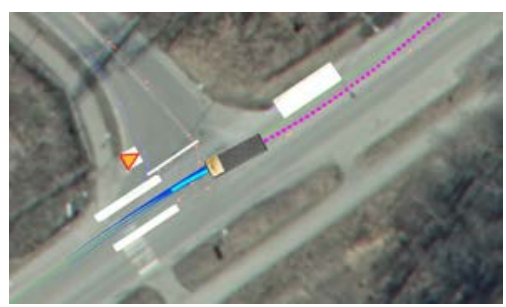

$v=3.6 \mathrm{~m} / \mathrm{s}$

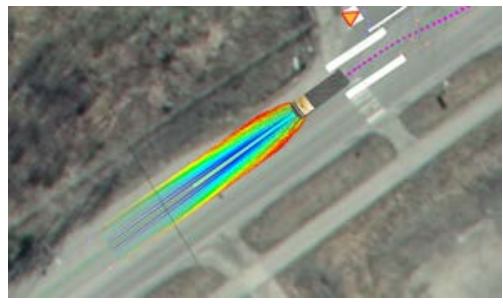

$v=5.4 \mathrm{~m} / \mathrm{s}$

Figure 3.11: A truck is approaching 3 obstacles shown as white boxes. The candidate trajectories are projected on the road surface and colored according to the cost $J[x(t)]$. Red means high cost and blue low cost. The selected path is shown as the thick green trajectory.

a behavior layer is often responsible for long term behavior and selects suitable terminal sets given the current traffic scene, so most computational effort is placed in interesting regions. However, independent of which trajectory generation algorithm that is used the hardest problem in structured areas is not to generate the trajectories themselves but to accurately predict the intentions of other traffic participants and account for this uncertainty within the planning problem and generate trajectories that feel natural and are predictable and acceptable to others that are interacting with the autonomous vehicle. Further complicating the prediction of other participants is the feedback generated from the interaction between the ego vehicle's decisions and the resulting actions of other participants and this interaction is investigated in Paper D. 


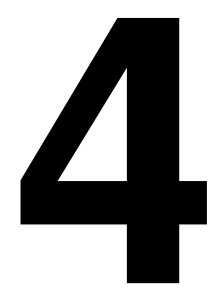

\section{Concluding remarks}

This chapter gives a summary of the contributions of the papers that constitute this thesis and discusses directions for future work. The first part of this thesis gave an introduction on some common techniques used to solve motion planning problems. These techniques have then been used and modified for a number of practical problems in both unstructured and structured areas and the results have been presented in the papers included in the thesis.

\subsection{Summary of contributions}

Paper A and D consider the kinodynamic planning problem for heavy duty trucks in scenarios where real-time aspects of algorithms are critical and a fast and responsive planner is needed. Paper B and C tackle non-holonomic planning problems for complicated vehicle models in unstructured areas where more deliberation time is given.

Paper A uses a probabilistic planner to handle a dynamically changing environment in an unstructured scenario which constantly requires re-planning when new information is received. The underlying algorithm is based on RRTs and have been modified to quickly rebuild and discard branches from the tree when new information is gained. The algorithm is called Closed Loop-RRT (CL-RRT) and was originally developed by Kuwata et al. (2009) for the DARPA Urban Challenge. In this paper an extension that significantly increases the probability of drawing a valid sample in critical situations is presented. A deterministic break profile calculation in the tree construction phase is introduced to calculate a new acceleration profile when a new branch has been found unfeasible. If possible, the new acceleration profile stops the vehicle before reaching the obstacle and a new extension can be simulated in the tree. The approach is tested on a heavy duty Scania G480 mining truck in a basic constructed scenario. 
In Paper $\mathrm{B}$ and $\mathrm{C}$ we consider stabilization and planning for a reversing off axle hitched truck with a dolly steered trailer. An RRT together with a path following pure pursuit controller is used to generate complex motions in unstructured environments that are tested on a small scale experimental platform with good results. No real-time constraints are imposed on the system and the system is intended as an advanced driver assistance system to help drivers with the complex task of reversing with trailers. To the author's knowledge this was the first planning framework considering the general 2-trailer configuration with off-axle hitch.

In Paper D we develop an algorithm for time critical scenarios in congested traffic. First we demonstrate the drawbacks of the commonly used constant velocity model for predictions of other road users and motivate the use of the Intelligent Driver Model (IDM) to build a prediction engine that tries to predict the development of the complete traffic scene given our own intended trajectory. A planning framework is then developed around the prediction engine where the induced acceleration on other participants given our own actions can be predicted and evaluated at planning time. In this way we get a parameter where the aggressiveness or politeness of our vehicle can be tuned. The planner is then demonstrated in a series of dense traffic situations for intersection and highway merging where interaction with other participants is necessary.

\subsection{Future work}

There is still a long road ahead before we see autonomous vehicles operating within our cities without any human supervision and many research questions remains. We will look at some possible directions for future work within each of the areas touched by the papers.

\section{Kinodynamic planning in unstructured areas}

Paper A used a simple model for the truck and focused on the calculation of a safe velocity profile that tried to ensure that a valid route to a complete stop was always available. An interesting direction for future work within a mining scenario is to use more advanced models that account for tip over by incorporating 3D information about the environment and extending the vehicle model to account for the center of mass together with roll and pitch angles. Due to the incremental nature of the RRT the implementation would be straightforward as long as it is possible to integrate the model forward in time.

So far only one vehicle has been considered, developing algorithms for coordination of multiple vehicles is also an interesting direction for future work.

\section{General 2-trailer}

The paths delivered by the RRT algorithm can sometimes be of questionable quality and include many unnecessary direction changes and turns. Investigation of fast optimization routines to solve the TPBVP so RRT* can be used and give probabilistic optimality would be interesting. If the TPBVP proves to be too time consuming an offline lattice could be constructed and a search based planner will probably give a better result. 
Full scale tests and an investigation of the effect on the control performance of estimation errors of the trailer angles would also be useful for further development.

\section{Structured planning}

Urban areas present an autonomous vehicle with an extremely complicated scene. Even if a perfect perception system would be available predicting future intentions and incorporating it within a planning framework that handles most of the, possibly infinite, situations that it can encounter is extremely hard. Developing algorithms or frameworks that incorporate the uncertainties from perception and prediction layers is a very interesting direction for future work.

Cost functions usually contain several different parameters that can be tuned and it is probably unlikely that one set of parameters can cover all situations. Investigating the feasibility of using unsupervised learning methods on data gathered while driving to construct a parameter set or cost function that is suitable for the current situation is also an interesting direction. 



\section{Bibliography}

Claudio Altafini, Alberto Speranzon, and Karl Henrik Johansson. Hybrid control of a truck and trailer vehicle. In Hybrid Systems: Computation and Control, pages 21-34. Springer, 2002.

R. Attia, R. Orjuela, and M. Basset. Combined longitudinal and lateral control for automated vehicle guidance. Vehicle System Dynamics, 52(2):261-279, 2014.

Jérôme Barraquand and J-C Latombe. A monte-carlo algorithm for path planning with many degrees of freedom. In Robotics and Automation, 1990. Proceedings., 1990 IEEE International Conference on, pages 1712-1717. IEEE, 1990.

Jerome Barraquand and Jean-Claude Latombe. Robot motion planning: A distributed representation approach. The International Journal of Robotics Research, 10(6):628649, 1991.

C. Beal and J. Gerdes. Model predictive control for vehicle stabilization at the limits of handling. IEEE Transactions on Control Systems Technology, 21(4):1258-1269, 2013.

John T Betts. Practical methods for optimal control and estimation using nonlinear programming, volume 19. Siam, 2010.

F. Borrelli, P. Falcone, and T. Keviczky. MPC-Based Approach to Active Steering for Autonomous Vehicle Systems. International Journal of Vehicle Autonomous Systems, 3(2):265-291, 2005.

Deborah Braid, Alberto Broggi, and Gary Schmiedel. The TerraMax autonomous vehicle. Journal of Field Robotics, 23(9):693-708, 2006.

Alberto Broggi, Massimo Bertozzi, Alessandra Fascioli, C Guarino Lo Bianco, and Aurelio Piazzi. The ARGO autonomous vehicle's vision and control systems. International Journal of Intelligent Control and Systems, 3(4):409-441, 1999.

Alberto Broggi, Pietro Cerri, Stefano Debattisti, Maria Chiara Laghi, Paolo Medici, Daniele Molinari, Matteo Panciroli, and Antonio Prioletti. PROUD-Public Road Urban Driverless-Car Test. IEEE Transactions on Intelligent Transportation Systems, 16 (6):3508-3519, 2015. 
Michael Calonder, Vincent Lepetit, Christoph Strecha, and Pascal Fua. BRIEF: Binary Robust Independent Elementary Features. In European conference on computer vision, pages 778-792. Springer, 2010.

Howie Choset, Kevin M. Lynch, Seth Hutchinson, George A Kantor, Wolfram Burgard, Lydia E. Kavraki, and Sebastian Thrun. Principles of Robot Motion: Theory, Algorithms, and Implementations. MIT Press, Cambridge, MA, June 2005.

Marcello Cirillo, Tansel Uras, and Sven Koenig. A lattice-based approach to multi-robot motion planning for non-holonomic vehicles. In 2014 IEEE/RSJ International Conference on Intelligent Robots and Systems, pages 232-239. IEEE, 2014.

Thomas H. Cormen, Charles E. Leiserson, Ronald L. Rivest, and Clifford Stein. Introduction to Algorithms. The MIT Press, 3 edition, 2009.

R. Coulter. Implementation of the pure-pursuit path tracking algorithm. Technical report, Carnegie Mellon University, 1992.

Alexander G Cunningham, Enric Galceran, Ryan M Eustice, and Edwin Olson. MPDM: Multipolicy decision-making in dynamic, uncertain environments for autonomous driving. In Robotics and Automation (ICRA), 2015 IEEE International Conference on, pages 1670-1677. IEEE, 2015.

Said Dahdah and Kate McMahon. The true cost of road crashes: Valuing life and the cost of a serious injury. International Road Assessment Programme, 2008.

Mark De Berg, Marc Van Kreveld, Mark Overmars, and Otfried Cheong Schwarzkopf. Computational Geometry: Algorithms and Applications. Springer, 3 edition, 2008.

Ernst Dieter Dickmanns and Alfred Zapp. A curvature-based scheme for improving road vehicle guidance by computer vision. In Cambridge Symposium_Intelligent Robotics Systems, pages 161-168. International Society for Optics and Photonics, 1987.

Ernst Dieter Dickmanns, Reinhold Behringer, Dirk Dickmanns, Thomas Hildebrandt, Markus Maurer, Frank Thomanek, and Joachim Schiehlen. The seeing passenger car VaMoRs-P. In Proceedings of the Intelligent Vehicles Symposium, pages 68-73. IEEE, October 1994.

Edsger W Dijkstra. A note on two problems in connexion with graphs. Numerische mathematik, 1(1):269-271, 1959.

Dmitri Dolgov, Sebastian Thrun, Michael Montemerlo, and James Diebel. Path planning for autonomous vehicles in unknown semi-structured environments. The International Journal of Robotics Research, 29(5):485-501, 2010.

Lester E Dubins. On curves of minimal length with a constraint on average curvature, and with prescribed initial and terminal positions and tangents. American Journal of mathematics, pages 497-516, 1957.

Julian Eggert, Florian Damerow, and Stefan Klingelschmitt. The foresighted driver model. In Intelligent Vehicles Symposium (IV), 2015 IEEE, pages 322-329. IEEE, 2015. 
A. Elfes. Using occupancy grids for mobile robot perception and navigation. Computer, 22(6):46-57, 1989.

European Comission. Roadmap to a single european transport area - towards a competitive and resource efficient transport system. Technical report, March 2011.

European Road Transport Research Advisory Council. Multi-annual implementation plan for horizon 2020. Technical report, March 2013.

Niclas Evestedt, Daniel Axehill, Marco Trincavelli, and Fredrik Gustafsson. Sampling Recovery for Closed Loop Rapidly Expanding Random Tree using Brake Profile Regeneration. In 2015 IEEE Intelligent Vehicles Symposium (IV), pages 101-106. IEEE, 2015 .

Niclas Evestedt, Oskar Ljungqvist, and Daniel Axehill. Motion planning for a reversing general 2-trailer configuration using Closed-Loop RRT. In 2016 IEEE/RSJ International Conference on Intelligent Robots and Systems (IROS), pages 3690 - 3697. IEEE, 2016a.

Niclas Evestedt, Oskar Ljungqvist, and Daniel Axehill. Path tracking and stabilization for a reversing general 2-trailer configuration using a cascaded control approach. In 2016 IEEE Intelligent Vehicles Symposium (IV), pages 1156 - 1161. IEEE, 2016b.

Niclas Evestedt, Erik Ward, John Folkesson, and Daniel Axehill. Interaction aware trajectory planning for merge scenarios in congested traffic situations. In 2016 IEEE Intelligent Transportation Systems Conference (ITSC), pages 465 - 472. IEEE, 2016c.

P. Falcone, F. Borrelli, E. Tseng, J. Asgari, and D. Hrovat. A hierarchical model predictive control framework for autonomous ground vehicles. In Proceedings of the American Control Conference, pages 3719-3724, June 2008.

Dave Ferguson and Anthony Stentz. Field D*: An interpolation-based path planner and replanner. In Robotics research, pages 239-253. Springer, 2007.

Dave Ferguson, Thomas M Howard, and Maxim Likhachev. Motion planning in urban environments. Journal of Field Robotics, 25(11-12):939-960, 2008.

Robert A. Ferlis. The Dream of an Automated Highway. US Department of Transportation, 71(1), 2007.

Ford. Ford First Automaker to Test Autonomous Vehicle at Mcity, University of Michigan's Simulated Urban Environment, 2015. URL https://media. ford.com/content/fordmedia/fna/us/en/news/2015/11/13/ ford-first-automaker-to-test-autonomous-vehicle-at-mcity . html.

Jerome H Friedman, Jon Louis Bentley, and Raphael Ari Finkel. An algorithm for finding best matches in logarithmic expected time. ACM Transactions on Mathematical Software (TOMS), 3(3):209-226, 1977. 
Jonathan D Gammell, Siddhartha S Srinivasa, and Timothy D Barfoot. Informed RRT*: Optimal sampling-based path planning focused via direct sampling of an admissible ellipsoidal heuristic. In 2014 IEEE/RSJ International Conference on Intelligent Robots and Systems.

Google. Google self-driving car project, 2016. URL https://www.google.com/ selfdrivingcar/.

Peter E Hart, Nils J Nilsson, and Bertram Raphael. A formal basis for the heuristic determination of minimum cost paths. IEEE transactions on Systems Science and Cybernetics, 4(2):100-107, 1968.

Boris Houska, Hans Joachim Ferreau, and Moritz Diehl. ACADO toolkit-An opensource framework for automatic control and dynamic optimization. Optimal Control Applications and Methods, 32(3):298-312, 2011.

Maciej Kalisiak and Michiel van de Panne. RRT-blossom: RRT with a Local Flood-fill Behavior. In ICRA, pages 1237-1242, 2006.

Sertac Karaman and Emilio Frazzoli. Incremental sampling-based algorithms for optimal motion planning. Robotics Science and Systems VI, 104, 2010a.

Sertac Karaman and Emilio Frazzoli. Optimal kinodynamic motion planning using incremental sampling-based methods. In 49th IEEE conference on decision and control (CDC), pages 7681-7687. IEEE, 2010b.

Sertac Karaman and Emilio Frazzoli. Sampling-based algorithms for optimal motion planning. The International Journal of Robotics Research, 30(7):846-894, 2011.

Takeo Kato, Yoshiki Ninomiya, and Ichiro Masaki. An obstacle detection method by fusion of radar and motion stereo. IEEE Transactions on Intelligent Transportation Systems, 3(3):182-188, 2002.

Lydia E Kavraki, Petr Svestka, J-C Latombe, and Mark H Overmars. Probabilistic roadmaps for path planning in high-dimensional configuration spaces. IEEE transactions on Robotics and Automation, 12(4):566-580, 1996.

Sven Koenig and Maxim Likhachev. D* Lite. In AAAI/IAAI, pages 476-483, 2002.

Alex Krizhevsky, Ilya Sutskever, and Geoffrey E Hinton. Imagenet classification with deep convolutional neural networks. In Advances in neural information processing systems, pages 1097-1105, 2012.

Tobias Kunz and Mike Stilman. Kinodynamic RRTs with fixed time step and best-input extension are not probabilistically complete. In Algorithmic Foundations of Robotics XI, pages 233-244. Springer, 2015.

Yoshiaki Kuwata, Justin Teo, Gaston Fiore, Sertac Karaman, Emilio Frazzoli, and Jonathan P How. Real-time motion planning with applications to autonomous urban driving. IEEE Transactions on Control Systems Technology, 17(5):1105-1118, 2009. 
Henning Lategahn and Christoph Stiller. Vision-only localization. IEEE Transactions on Intelligent Transportation Systems, 15(3):1246-1257, 2014.

Jean-Claude Latombe. Robot motion planning, volume 124. Springer Science \& Business Media, 2012.

Steven M LaValle. Rapidly-exploring random trees: A new tool for path planning. 1998.

Steven M LaValle. Planning algorithms. Cambridge university press, 2006.

Steven M LaValle and James J Kuffner. Randomized kinodynamic planning. The International Journal of Robotics Research, 20(5):378-400, 2001.

Steven M. LaValle, James J. Kuffner, and Jr. Rapidly-exploring random trees: Progress and prospects, 2000.

Anders Lie, Claes Tingvall, Maria Krafft, and Anders Kullgren. The effectiveness of electronic stability control (ESC) in reducing real life crashes and injuries. Traffic injury prevention, 7(1):38-43, 2006.

Martin Liebner, Felix Klanner, Michael Baumann, Christian Ruhhammer, and Christoph Stiller. Velocity-based driver intent inference at urban intersections in the presence of preceding vehicles. Intelligent Transportation Systems Magazine, IEEE, 5(2):10-21, 2013.

Maxim Likhachev, Geoffrey J Gordon, and Sebastian Thrun. ARA*: Anytime A* with provable bounds on sub-optimality. In Advances in Neural Information Processing Systems, page None, 2003.

Maxim Likhachev, David I Ferguson, Geoffrey J Gordon, Anthony Stentz, and Sebastian Thrun. Anytime Dynamic A*: An Anytime, Replanning Algorithm. In ICAPS, pages 262-271, 2005.

P. Lima, M. Trincavelli, J. Mårtensson, M. Nilsson, and B. Wahlberg. Experimental evaluation of predictive lateral control for an autonomous truck. In Proceedings of the IEEE Intelligent Vehicles Symposium, June 2016.

Jonathan Long, Evan Shelhamer, and Trevor Darrell. Fully convolutional networks for semantic segmentation. In Proceedings of the IEEE Conference on Computer Vision and Pattern Recognition, pages 3431-3440, 2015.

David G Lowe. Object recognition from local scale-invariant features. In Computer vision, 1999. The proceedings of the seventh IEEE international conference on, volume 2, pages 1150-1157. Ieee, 1999.

Tomas Lozano-Perez. Spatial planning: A configuration space approach. IEEE transactions on computers, 100(2):108-120, 1983.

Malin Lundgren, Erik Stenborg, Lennart Svensson, and Lars Hammarstrand. Vehicle self-localization using off-the-shelf sensors and a detailed map. In Intelligent Vehicles Symposium Proceedings, 2014 IEEE, pages 522-528. IEEE, 2014. 
David Madås, Mohsen Nosratinia, Mansour Keshavarz, Peter Sundström, Rolland Philippsen, Andreas Eidehall, and Karl-Magnus Dahlén. On path planning methods for automotive collision avoidance. In Intelligent Vehicles Symposium (IV), 2013 IEEE, pages 931-937. IEEE, 2013.

Molly Mchug. Tesla's cars now drive themselves, kinda,
2015.
URL

Colin McManus, Winston Churchill, Will Maddern, Alexander D Stewart, and Paul Newman. Shady dealings: Robust, long-term visual localisation using illumination invariance. In 2014 IEEE International Conference on Robotics and Automation (ICRA), pages 901-906. IEEE, 2014.

Michael Montemerlo, Jan Becker, Suhrid Bhat, Hendrik Dahlkamp, Dmitri Dolgov, Scott Ettinger, Dirk Haehnel, Tim Hilden, Gabe Hoffmann, Burkhard Huhnke, et al. Junior: The stanford entry in the urban challenge. Journal of Field Robotics, 25(9):569-597, 2008 .

Hans P Moravec. The Stanford cart and the CMU rover. In Autonomous Robot Vehicles, pages 407-419. Springer, 1990.

Malte Moritz and Anton Pettersson. Estimation of Local Map from Radar Data. Master's thesis, Linköping University, 2014.

David Z. Morris. Today's cars are parked 95\% of the time, 2016. URL http: //fortune.com/2016/03/13/cars-parked-95-percent-of-time.

Jauwairia Nasir, Fahad Islam, Usman Malik, Yasar Ayaz, Osman Hasan, Mushtaq Khan, and Mannan Saeed Muhammad. RRT*-SMART: A rapid convergence implementation of RRT. International Journal of Advanced Robotic Systems, 10, 2013.

National Highway Traffic Safety Administration. Auto Industry Commitment to IIHS and NHTSA on Automatic Emergency Braking. Technical report, March 2015a.

National Highway Traffic Safety Administration. Critical reasons for crashes investigated in the national motor vehicle crash causation survey. Technical report, February $2015 \mathrm{~b}$.

Jorge Nocedal and Stephen Wright J. Numerical Optimization. Springer New York, 2006.

Tobias Nothdurft, Peter Hecker, Sebastian Ohl, Falko Saust, Markus Maurer, Andreas Reschka, and Jürgen Rüdiger Böhmer. Stadtpilot: First fully autonomous test drives in urban traffic. In 2011 14th International IEEE Conference on Intelligent Transportation Systems (ITSC), pages 919-924. IEEE, 2011.

Mihail Pivtoraiko, Ross A Knepper, and Alonzo Kelly. Differentially constrained mobile robot motion planning in state lattices. Journal of Field Robotics, 26(3):308-333, 2009.

James Reeds and Lawrence Shepp. Optimal paths for a car that goes both forwards and backwards. Pacific journal of mathematics, 145(2):367-393, 1990. 
Julian Schlechtriemen, Florian Wirthmueller, Andreas Wedel, Gabi Breuel, and KlausDieter Kuhnert. When will it change the lane? A probabilistic regression approach for rarely occurring events. In 2015 IEEE Intelligent Vehicles Symposium (IV), pages 1373-1379. IEEE, 2015.

Lukas Schneider, Marius Cordts, Timo Rehfeld, David Pfeiffer, Markus Enzweiler, Uwe Franke, Marc Pollefeys, and Stefan Roth. Semantic stixels: Depth is not enough. In Proceedings of the 2016 IEEE Intelligent Vehicles Symposium. IEEE, 2016.

Markus Schreiber, Carsten Knöppel, and Uwe Franke. Laneloc: Lane marking based localization using highly accurate maps. In Intelligent Vehicles Symposium (IV), 2013 IEEE, pages 449-454. IEEE, 2013.

Ulrich Schwesinger, Martin Rufli, Paul Furgale, and Roland Siegwart. A sampling-based partial motion planning framework for system-compliant navigation along a reference path. In Intelligent Vehicles Symposium (IV), 2013 IEEE, pages 391-396. IEEE, 2013.

Anthony Stentz. Optimal and efficient path planning for partially-known environments. In Robotics and Automation, 1994. Proceedings., 1994 IEEE International Conference on, pages 3310-3317. IEEE, 1994.

Subh83. Astar progress animation, a. URL https://commons.wikimedia. org/wiki/File:Astar_progress_animation.gif. Modified under Creative Commons license.

Subh83. Dijkstras progress animation, b. URL https://commons.wikimedia. org/wiki/File:Dijkstras_progress_animation.gif. Modified under Creative Commons license.

Charles Thorpe, Martial H Hebert, Takeo Kanade, and Steven A Shafer. Vision and navigation for the Carnegie-Mellon Navlab. IEEE Transactions on Pattern Analysis and Machine Intelligence, 10(3):362-373, 1988.

Charles Thorpe, Martial Herbert, Takeo Kanade, and Steven Shafer. Toward autonomous driving: The CMU Navlab Part I - Perception. IEEE expert, 6(4):31-42, 1991.

Sebastian Thrun, Mike Montemerlo, Hendrik Dahlkamp, David Stavens, Andrei Aron, James Diebel, Philip Fong, John Gale, Morgan Halpenny, Gabriel Hoffmann, et al. Stanley: The robot that won the DARPA Grand Challenge. Journal of Field Robotics, 23(9):661-692, 2006.

Paul G Trepagnier, Powell M Kinney, Jorge E Nagel, Matthew T Dooner, and Jason S Pearce. Team Gray technical paper. DARPA Grand Challenge, 2005.

Shriram Mahabal Udupa. Collision detection and avoidance in computer controlled manipulators. PhD thesis, California Institute of Technology, 1977.

Chris Urmson and Reid G Simmons. Approaches for heuristically biasing RRT growth. In IROS, volume 2, pages 1178-1183, 2003. 
Chris Urmson, Joshua Anhalt, Drew Bagnell, Christopher Baker, Robert Bittner, MN Clark, John Dolan, Dave Duggins, Tugrul Galatali, Chris Geyer, et al. Autonomous driving in urban environments: Boss and the urban challenge. Journal of Field Robotics, 25(8):425-466, 2008.

HDL-64E S3 User Manual. Velodyne Inc, May 2013. Rev G.

Dominic Zeng Wang, Ingmar Posner, and Paul Newman. What could move? Finding cars, pedestrians and bicyclists in 3D laser data. In Robotics and Automation (ICRA), 2012 IEEE International Conference on, pages 4038-4044. IEEE, 2012.

E. Ward and J. Folkesson. Multi-classification of driver intentions in yielding scenarios. In Proceedings of the International IEEE Intelligent Transportation Systems Conference, pages 678-685, September 2015.

Erik Ward and John Folkesson. Vehicle localization with low cost radar sensors. In Proceedings of the 2016 IEEE Intelligent Vehicles Symposium. IEEE, 2016.

Junqing Wei, John M Dolan, and Bakhtiar Litkouhi. Autonomous vehicle social behavior for highway entrance ramp management. In Intelligent Vehicles Symposium (IV), 2013 IEEE, pages 201-207. IEEE, 2013.

Moritz Werling, Tobias Gindele, Daniel Jagszent, and Lutz Gröll. A robust algorithm for handling moving traffic in urban scenarios. In Intelligent Vehicles Symposium, 2008 IEEE, pages 1108-1112. IEEE, 2008.

Moritz Werling, Sören Kammel, Julius Ziegler, and Lutz Gröll. Optimal trajectories for time-critical street scenarios using discretized terminal manifolds. The International Journal of Robotics Research, 31(3):346-359, 2011.

William Whittaker. Red Team DARPA Grand Challenge Technical Paper. Technical report, August 2005.

Ryan W. Wolcott and Ryan M. Eustice. Visual localization within LIDAR maps for automated urban driving. In Proceedings of the IEEE/RSJ International Conference on Intelligent Robots and Systems, pages 176-183, Chicago, IL, USA, September 2014.

Ryan W. Wolcott and Ryan M. Eustice. Fast LIDAR localization using multiresolution Gaussian mixture maps. In Proceedings of the IEEE International Conference on Robotics and Automation, pages 2814-2821, Seattle, WA, USA, May 2015.

World Health Organization. Global status report on road safety. Technical report, October 2015.

Julius Ziegler, Philipp Bender, Thao Dang, and Christoph Stiller. Trajectory planning for Bertha-a local, continuous method. In 2014 IEEE Intelligent Vehicles Symposium Proceedings, pages 450-457. IEEE, 2014a.

Julius Ziegler, Philipp Bender, Markus Schreiber, Henning Lategahn, Tobias Strauss, Christoph Stiller, Thao Dang, Uwe Franke, Nils Appenrodt, Christoph G Keller, et al. Making bertha drive-An autonomous journey on a historic route. IEEE Intelligent Transportation Systems Magazine, 6(2):8-20, 2014b. 
Part II

\section{Publications}





\section{Papers}

The articles associated with this thesis have been removed for copyright reasons. For more details about these see:

http://urn.kb.se/resolve?urn=urn:nbn:se:liu:diva-132769 